\title{
A Material Point Method for Thin Shells with Frictional Contact
}

\author{
QI GUO, University of California, Los Angeles \\ XUCHEN HAN, University of California, Los Angeles \\ CHUYUAN FU, University of California, Los Angeles \\ THEODORE GAST, University of California, Los Angeles \\ RASMUS TAMSTORF, Walt Disney Animation Studios \\ JOSEPH TERAN, University of California, Los Angeles
}

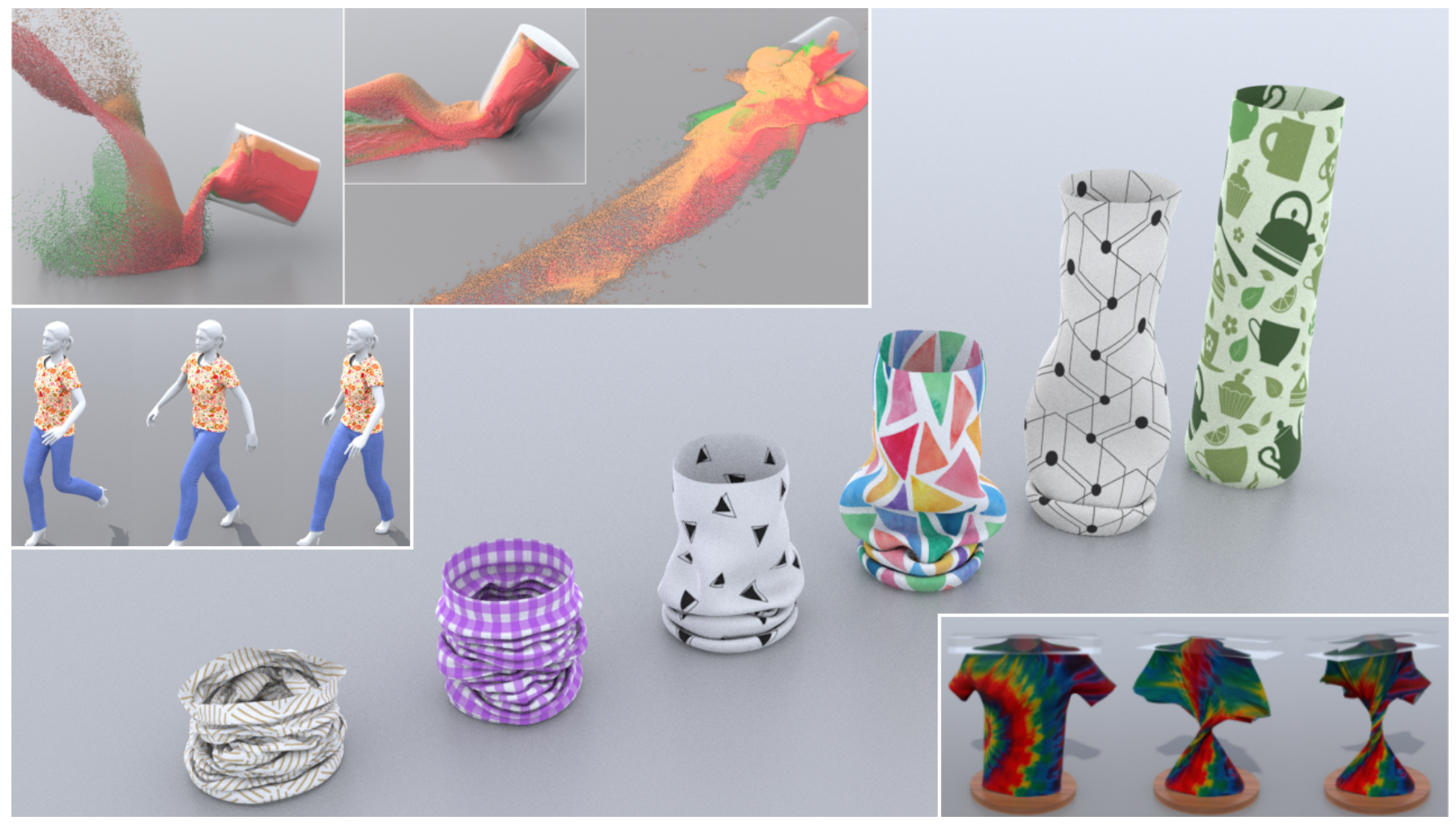

Fig. 1. Shell Montage. Upper left : simulation of shells coupled with granular materials. Center left : a walk cycle benchmark for clothing simulation. Bottom right : a T-shirt twisted to induce many self-collisions. Center : the effect of increasing bending stiffness (from left to right) for six collapsing elastic cylinders.

Authors' addresses: Qi Guo, University of California, Los Angeles, qiguo@ucla.edu; Xuchen Han, University of California, Los Angeles, xhan0619@ucla.edu; Chuyuan Fu, University of California, Los Angeles, fuchuyuan@math.ucla.edu; Theodore Gast, University of California, Los Angeles, tfg@math.ucla.edu; Rasmus Tamstorf, Walt Disney Animation Studios, rasmus.tamstorf@disneyanimation.com; Joseph Teran, University of California, Los Angeles, jteran@math.ucla.edu.

Permission to make digital or hard copies of all or part of this work for personal or classroom use is granted without fee provided that copies are not made or distributed for profit or commercial advantage and that copies bear this notice and the full citation on the first page. Copyrights for components of this work owned by others than the author(s) must be honored. Abstracting with credit is permitted. To copy otherwise, or republish, to post on servers or to redistribute to lists, requires prior specific permission and/or a fee. Request permissions from permissions@acm.org.

(C) 2018 Copyright held by the owner/author(s). Publication rights licensed to Association for Computing Machinery.

0730-0301/2018/8-ART147 \$15.00

https://doi.org/10.1145/3197517.3201346
We present a novel method for simulation of thin shells with frictional contact using a combination of the Material Point Method (MPM) and subdivision finite elements. The shell kinematics are assumed to follow a continuum shell model which is decomposed into a Kirchhoff-Love motion that rotates the mid-surface normals followed by shearing and compression/extension of the material along the mid-surface normal. We use this decomposition to design an elastoplastic constitutive model to resolve frictional contact by decoupling resistance to contact and shearing from the bending resistance components of stress. We show that by resolving frictional contact with a continuum approach, our hybrid Lagrangian/Eulerian approach is capable of simulating challenging shell contact scenarios with hundreds of thousands to millions of degrees of freedom. Without the need for collision detection or resolution, our method runs in a few minutes per frame in these high resolution examples. Furthermore we show that our technique naturally couples with other traditional MPM methods for simulating granular and related materials. 


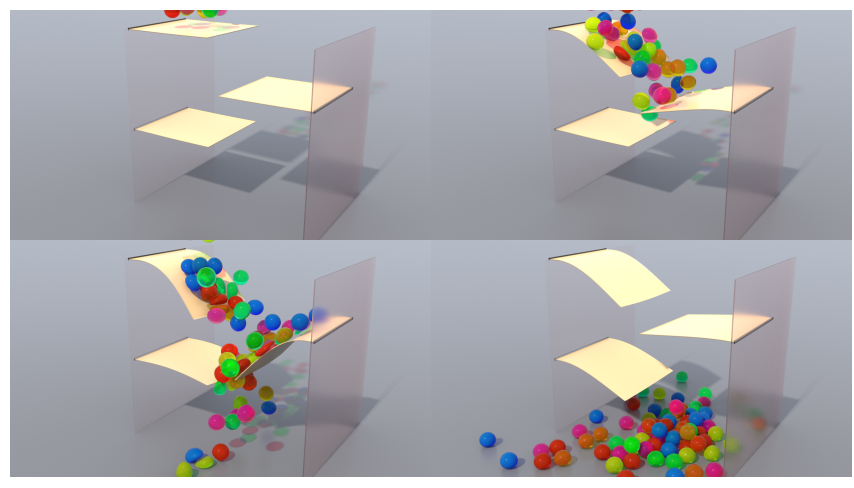

Fig. 2. Elastic spheres on diving boards. We demonstrate appealing dynamics achieved with self-collision and appreciable bending for shells. Both the spheres and the diving boards are simulated as thin shells.

\section{CCS Concepts: • Computing methodologies $\rightarrow$ Physical simulation;}

Additional Key Words and Phrases: MPM, Elasticity, Thin shells, Cloth, Subdivision finite elements

\section{ACM Reference Format:}

Qi Guo, Xuchen Han, Chuyuan Fu, Theodore Gast, Rasmus Tamstorf, and Joseph Teran. 2018. A Material Point Method for Thin Shells with Frictional Contact. ACM Trans. Graph. 37, 4, Article 147 (August 2018), 15 pages. https: //doi.org/10.1145/3197517.3201346

\section{INTRODUCTION}

Simulation of thin elastic surfaces with bending resistance and frictional contact is essential in many domains including visual effects, textile simulation, as well as engineering applications that involve thin metallic sheets. The most common computer graphics techniques for simulating these materials typically use mass/spring models or continuum elastic formulations over linear strain triangles. The popularity of these approaches is primarily due to their relative simplicity, computational efficiency and the favorable performance of contact/collision techniques. However, these techniques have well-known limitations that include resolution dependent behavior like mesh-based anisotropy and lack of convergence under refinement for mass/spring models. Furthermore, constitutive models for bending resistance can be difficult to discretize over triangle meshes since higher order derivatives needed for curvature calculations are not easily approximated with linear interpolation. In contrast, shell models commonly used in the engineering literature provide natural and accurate control of the constitutive behavior caused by bending and converge under refinement due to their continuum foundations. However, these models typically require $H^{2}$ regularity that is difficult to achieve with traditional interpolation. The $H^{2}$ regularity requirement means that interpolating functions and all their derivatives of order less than or equal to two are square integrable. In practice this means that the interpolating functions must also have continuous first derivatives ( $C^{1}$ continuous). Non-uniform rational B-splines (NURBS) [2014] and subdivision (subd) surface interpolation [2000] yield the required regularity, but these more elaborate interpolation strategies can complicate collision detection and resolution. Thus with existing methods, the choice is between on one hand using linear triangles and getting natural resolution of collisions and contact but having limited constitutive control and on the other hand using shell-based techniques with better constitutive control at the expense of more complicated collision and contact.

We develop a continuum shell approach that achieves both natural constitutive control and efficient and robust collision and contact resolution. Historically, the difficulties with shell-based collisions arise from the purely Lagrangian nature of commonly used techniques. It is more difficult to impose and resolve collision constraints over subd and NURBS surfaces than it is over triangle meshes with linear interpolation (see Eischen et al.[1996], Thomaszewski et al.[2006], and $\mathrm{Lu}$ and Zhang[2014]). Recently, approaches that utilize a hybrid Lagrangian/Eulerian view of the physics have been developed for collision and contact with elastic solids [Fan et al. 2013, 2014; Levin et al. 2011; Li et al. 2013; McAdams et al. 2009]. While Lagrangian methods require separate modeling for collision/contact resolution, Eulerian methods naturally resolve collision and contact through the elasticity physics. Notably, the hybrid Lagrangian/Eulerian collision formulation technique of Jiang et al.[2017] naturally resolves contact through an elastoplastic view of frictional contact with thin membranes. Jiang et al.[2017] showed that by letting the elastoplastic constitutive model resolve frictional contact through a continuum view, there is no need for collision detection or resolution via impulses [Bridson et al. 2002; Harmon et al. 2008; Sifakis et al. 2008; Tang et al. 2016] or linear complementary formulations of the constrained dynamics [Otaduy et al. 2009]. This greatly accelerates the performance since these routines are often a bottleneck. We generalize the elastoplastic frictional contact model of Jiang et al.[2017] from thin membranes and linear triangle meshes with limited bending resistance to volumetric shells discretized by subd finite elements capable of expressing bending resistance through Kirchhoff-Love continuum theory.

The Jiang et al. [2017] approach was designed for thin membranes with no resistance to bending. There is no mechanism for expressing resistance to bending in their formulation. It is possible to simply add bending springs to their approach, however, that violates the key assumptions in their elastoplastic characterization of frictional contact. This suffices for moderate resistance to bending. However, when visibly significant resistance to bending is required (e.g. for a stiff leather or denim garment etc), artifacts are apparent. We show that the Jiang et al. [2017] formulation can be generalized to shell model kinematics and that this in turn allows for the simulation of much larger ranges of bending stiffness without the artifacts inherent in ignoring bending related stress in elastoplastic continuum model.

Borrowing from the physics of frictional contact in granular materials [Klár et al. 2016], Jiang et al.[2017] imagine membranes as existing in a volumetric continuum where stresses resist not only deformation in the membrane but also compression and shearing normal to the membrane. It is these extra stress components that resolve membrane self-collision and contact. This view of the membrane as a thickened continuum is similar to what we propose here for shells. Jiang et al.[2017] track the deformation normal to the membrane on each triangle in the membrane mesh, which is very similar to Mindlin-Reissner[1951] or continuum shell kinematics. A Mindlin-Reissner shell is defined as a volumetric region obtained 


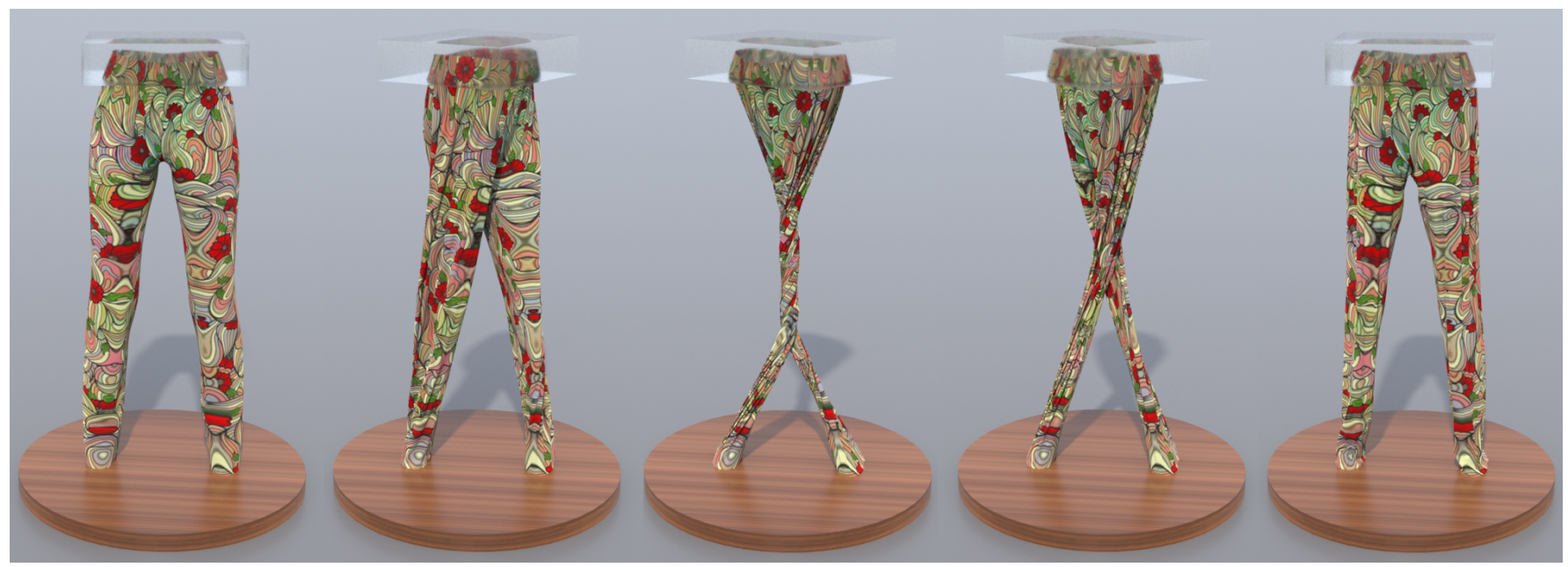

Fig. 3. Pants twister. Our approach works for clothing simulation with many self-collisions as shown here in the legs of a twisted pair of pants. The subdivision mesh for the pants has $393 \mathrm{~K}$ control points and the simulation runs at $78 \mathrm{~s}$ per frame.

by extruding a mid-surface in lines initially normal to it. These lines are often referred to as fibers [Belytschko et al. 2013]. The fibers represent material directions that remain linear through the shell kinematics (see Figure 7). In this view, the shell kinematics are determined from the motion of the mid-surface and the rotation and extension/compression of fibers normal to the mid-surface. In the Mindlin-Reissner kinematics, the fibers remain the same length Jiang et al. allow the fibers to stretch and compress, indeed this is essential in their formulation of contact penalty stress. The kinematics when the fibers are allowed to change length is referred to as a continuum shell assumption.

Given the similarities between the approach of Jiang et al.[2017] and the continuum shell kinematics, we combine them to define both bending resistant stresses and frictional contact stresses in the same constitutive model. In our approach, we decompose the continuum shell kinematics into two components to achieve this. The first component moves the mid-surface but does not allow shearing or compression in the fiber directions. The second component of the motion fixes the mid-surface but allows compression/extension and shearing of the fiber directions needed to define a general continuum shell motion. This splitting allows us to design a model that naturally decouples the response to frictional contact from the resistance to bending in a manner analogous to deviatoric/dilation splitting techniques commonly used for nearly incompressible elastic materials [Bonet and Wood 2008]. This decoupling is necessary to prevent the state of bending from affecting the frictional contact behavior and vice versa.

The motion in the first stage is often referred to as Kirchhoff-Love kinematics. By design, this motion does not see shearing or compression in the fiber directions and thus constitutive models with Kirchhoff-Love kinematics satisfy a zero transverse normal stress condition. This stress resistance to deformation in the internal surfaces above and below the mid-surface (often referred to as laminae [Belytschko et al. 2013]) creates resistance to mid-surface bending. However, the state of zero transverse normal stress in this model precludes resistance to contact normal to the mid-surface. The motion in the second stage accounts for the compression/extension of the material in the fiber directions and it is penalized as in Jiang et al.[2017]. Our splitting also allows us to formulate denting and wrinkling behaviors as plastic deformation in the Kirchhoff-Love component of the motion as in approaches like Gingold et al.[2004] and Narain et al.[2013].

We demonstrate the efficacy of our approach with a number of challenging simulations for shell and clothing simulation applications with scenarios involving hundreds of thousands to millions of degrees of freedom. Without the need for collision detection or resolution, we show that our method runs in a few minutes per frame in these high resolution examples. We summarize our novel contributions as

- An elastoplastic formulation for frictional contact and resistance to bending and denting of thin shells

- A strain splitting technique to separate thin shell motion into Kirchhoff-Love and continuum shell components

- A plane strain/stress formulation for Kirchhoff-Love thin shells that simplifies the return mapping algorithm for denting resistance

- A hybrid/Eulerian MPM discretization of the deformation gradient in the shell and the associated potential energy

\section{PREVIOUS WORK}

The literature in graphics and engineering related to simulation of clothing is extremely vast. Here we only discuss the work most related to our continuum based bending and collision models.

\subsection{Early continuum models}

Continuum models for elastic surfaces with bending resistance have been used for many years. Early methods had many limitations related to the treatment of self-collision, general mesh geometry/topology etc., but they demonstrated great promise and 


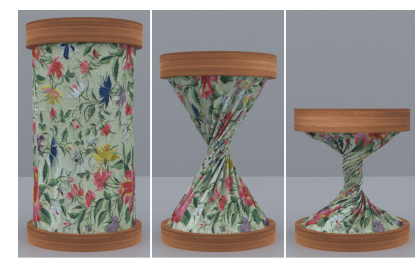

(a)

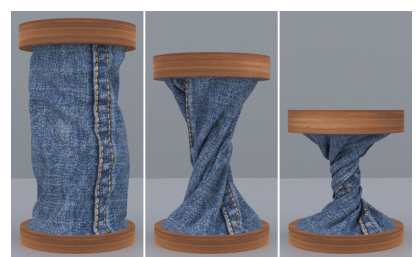

(b)
Fig. 4. Twisting Orthotropic Model. Using the data-driven model of Clyde et al. [2017] for woven materials, the characteristic wrinkling of silk (left) and denim (right) is obtained. Our method naturally resolves the many self-collisions induced by the twisting boundary conditions.

addressed aspects of the functionality we provide with our method. As an example, Terzopoulos et al.[1987] use the second fundamental form to define a bending energy and use finite differences to discretize the problem over a regular grid. Eischen et al.[1996] use a finite element method (FEM) discretization of Mindlin-Reissner shells to model quasistatic equilibrium with draping cloth. They use penalty methods for collision with external objects, but they do not handle self-collision. Other early works use continuum shell models like those of Simo et al.[1989] successfully for fabrics, albeit with limited support for self-collision [Chen and Govindaraj 1995; Collier et al. 1991; Gan et al. 1995; Man and Swan 2007]. Etzmuss et al.[2003a; 2003b] go further by approximating the bending response in cloth warp and weft directions using a discrete projected Laplacian, but the Laplacian approach is limited to flat reference configurations.

\subsection{Kirchhoff-Love}

The Kirchhoff-Love model is one example of a continuum model used for thin shells. The formulation applies the simple kinematic assumption that lines normal to the shell mid-surface always remain normal as the shell is deformed. However, the kinematic assumption requires higher order derivatives in the associated PDEs and this requires comparatively burdensome regularity of interpolation functions used in FEM calculations. Many approaches in engineering and graphics applications use Kirchhoff-Love continuum shells despite the additional regularity requirements. However, very few of them address the problem of self and external object collision. Cirak et al.[2000] use Loop's subdivision scheme for triangle meshes to develop FEM basis functions that are $H^{2}$ as required by KirchhoffLove theory for thin shells. Similarly, Wawrzinek et al.[2011] use the Catmull-Clark subdivision scheme and Lu and Zheng [2014] use the NURBS isogeometric analysis discretization of Kirchhoff-Love shells from Kiendl et al.[2009; 2015]. Of these, only Lu and Zheng [2014] address self and external body collisions, and Wawrzinek et al.[2011] and Cirak et al.[2000] only focus on linear elasticity and small strain problems. Cirak and Ortiz[2001] provide an extension of the subdivision approach in [2000] to allow for large deformation and nonlinear elasticity. A number of works including Thomaszewski et al.[2006], Grinspun et al.[1999] and Kaufmann et al.[2009] provide extensions to include inertia effects, implicit time stepping and large deformations. Kaufmann et al.[2009] use the Discontinous Galerkin (DG) approach of [Noels and Radovitzky 2008] to remove the need for $H^{2}$ interpolation. However, the DG approach requires the duplication of grid nodes on element boundaries which increases computational expense. Martin et al.[Martin et al. 2010] use Kirchhoff-Love shell and Kirchhoff rod models as motivation in their construction of a unified approach to simulation of elastic volumes, solids and rods. Clyde et al.[2017] design a new orthotropic hyperelastic constitutive model for Kirchhoff-Love shells simulated with subd FEM to fit experimental data, but they also do not provide a treatment for self-collision. Grinspun et al.[2002] develop an adaptive version of the subd interpolation functions. Similar to our approach, Long et al. [2012] show that shear-flexible shells can be decomposed into Kirchhoff-Love and shear motions. Remarkably they show that the splitting has no compatibility constraints on the shape functions used for discretizing the mid-surface and the shear vectors respectively. Echter et al. [Echter et al. 2013] use a family of isogeometric shell finite elements based on NURBS shape functions to satisfy the $H^{2}$ regularity requirements. Furthermore, as with our approach, they split the shell kinematics into bending and shear deformations and show that this results in an element that prevents shear locking for Mindlin-Reissner shell kinematics.

\subsection{Shell contact and collision}

Collision and contact handling for subd and NURBS based interpolation is challenging because of wider coupling between discrete degrees of freedom; however, added regularity in the surface can simplify some aspects [Matzen and Bischoff 2016; Matzen et al. 2013]. A detailed review of contact with isogeometric approaches for volumetric objects are provided in Temizer et al.[2011] and Lorentis et al.[2014]. Martin et al.[Martin et al. 2010] use forces derived from an energy that penalizes overlap of particles. Our collision stress response similarly arises from a potential, however, their approach is purely Lagrangian whereas ours is hybrid Lagrange/Eulerian. A number of works including Lu and Zheng [2014] and Thomaszewski et al.[2006] use the Bridson et al. impulse based approach for self-collision. However, the Bridson et al. [2002] approach to self-collision is designed for linear strain triangle meshes, this makes their application to more general meshes using subd and NURBS more challenging. Specifically, a triangulated mesh must be created solely for collision purposes and the application of the impulse can only be applied assuming linear interpolation, which is inaccurate. Lu and Zheng [2014] use the NURBS isogeometric analysis discretization of Kirchhoff-Love shells in Kiendl et al.[2009; 2015]. They use collision detection techniques from $\mathrm{Lu}$ [2011] and Temizer et al.[2011]. Grinspun et al.[1999] use a variational approach to selfcollision that is rooted in the approach of Kane et al.[1999] and the subdivision-surface interference detection algorithm in Grinspun and Schröder[2001]. Kane et al. [1999] use non-smooth analysis to formulate self-collision in a Newmark (implicit/explicit) time stepping schemes as nonlinearly constrained optimization problems which they solve with sequential quadratic programming (SQP). However, the approach in Kane et al. [1999] is computationally burdensome for simulations with moderate to high spatial mesh 
resolution.

\subsection{Lagrangian/Eulerian collision/contact}

A number of recent approaches have used hybrid Lagrangian and Eulerian views to simplify collision and contact treatment. Examples include simulation of elastoplastic solids using an Eulerian view of the governing physics [Fan et al. 2013, 2014; Levin et al. 2011; Li et al. 2013], using MPM [Daviet and Bertails-Descoubes 2016; Klár et al. 2016; Narain et al. 2010; Stomakhin et al. 2013; Yue et al. 2015; Zhu and Bridson 2005] and using Particle-In-Cell [McAdams et al. 2009]. Other hybrid approaches have been used successfully for solid/fluid coupling [Jiang et al. 2017, 2015; Teng et al. 2016] and for crowds [Golas et al. 2014; Narain et al. 2009]. Hybrid approaches of this type provide the starting point for our approach.

\subsection{Plasticity for wrinkles}

Our approach naturally supports plasticity based denting of shells. Similar approaches in the literature include that of Gingold et al.[2004] where they use a von-Mises yield condition with kinematic hardening to create denting and wrinkling effects. Narain et al.[2013] develop an adaptive method for triangle meshes to simulate detailed folds and wrinkles. They use the hinge bending models in Grinspun et al.[2003] and Bridson et al.[2003] with nonzero rest angles derived from the plasticity formulation in Gingold et al.[2004] Our plasticity approach for producing wrinkling behavior is very similar to these. However because we also use plasticity to model frictional contact, we design a decoupled plasticity model.

\section{OVERVIEW}

We first discuss our notation as well as related mathematical details (§4). We then outline the kinematic details of continuum shells and our splitting into Kirchhoff-Love and shearing/compression modes (§5). Next, we describe our continuous elastoplasticity model in terms of the potential energy density in the shell and its relation to plasticity constraints and associated return mappings for frictional contact as well as wrinkling and denting (§6). We then discuss subd FEM discretization of the potential energy and the derivatives of the energy with respect to discrete degrees of freedom (§9). Lastly we show that, as with many models defined in terms of an elastic potential [Jiang et al. 2017, 2015; Stomakhin et al. 2013], it is straightforward to discretize our model with MPM (§10).

\section{MATHEMATICAL DETAILS AND NOTATION}

We use bold face (e.g. v) to denote vector and tensor quantities and plain text (e.g. $v$ ) to denote scalar quantities. We use brackets around bold face to denote matrices associated with a tensor in a given basis (e.g $[\mathbf{M}] \in \mathbb{R}^{3 \times 3}$ is the matrix of entries $m_{i j} \in \mathbb{R}$ where tensor $\left.\mathbf{m}=m_{i j} \mathbf{e}_{i} \otimes \mathbf{e}_{j}\right)$. We use the convention that Greek indices (e.g. $a_{\alpha}$ ) range from $1-2$ and Latin indices (e.g. $b_{i}$ ) range from $1-3$. We use hat notation to indicate the upper left $2 \times 2 \mathrm{sub}$ matrix of a given matrix (e.g. $[\hat{\mathbf{M}}] \in \mathbb{R}^{2 \times 2}$ consists of entries $m_{\alpha \beta}$ from $[\mathbf{M}] \in \mathbb{R}^{3 \times 3}$ ). Unless otherwise stated, we use the summation convention for repeated indices. For a set of (covariant) basis vectors $\mathbf{v}_{i}$, we use $\mathbf{v}^{j}$ to denote the corresponding contravariant basis vectors satisfying $\mathbf{v}_{i} \cdot \mathbf{v}^{j}=\delta_{i}^{j} \cdot|\cdot|$ is used to denote the $L_{2}$ norm of a vector.

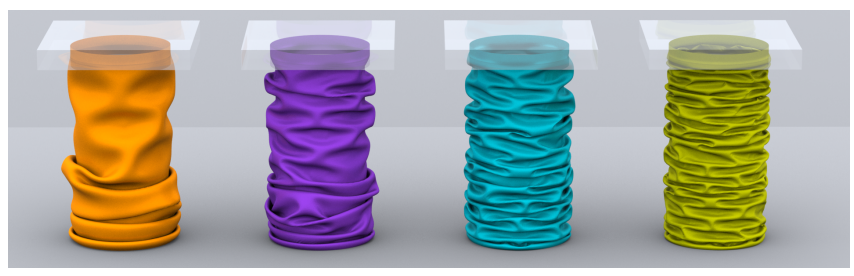

Fig. 5. Variation in shell thickness. We demonstrate the effect of the shell thickness parameter in a compression comparison.
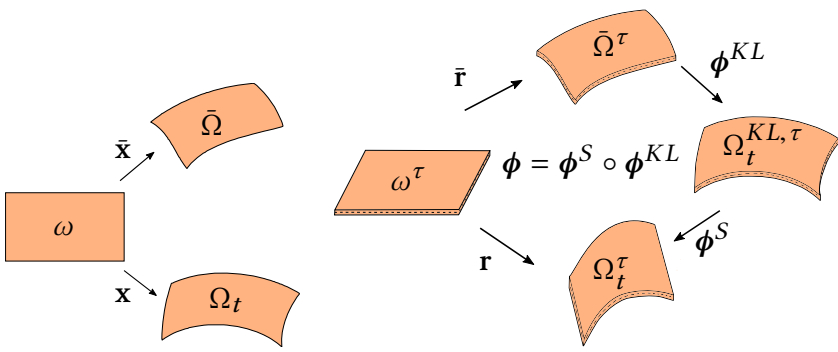

Fig. 6. Shell Kinematics. On the left, the mid-surface mappings are illustrated, and on the right the corresponding volumetric shell mappings are shown.

We assume shells have constant thickness $\tau$ and use $\omega^{\tau}=\omega \times$ $\left[-\frac{\tau}{2}, \frac{\tau}{2}\right]$ to parameterize the domain of the shell where $\omega$ is twodimensional parameter domain for the mid-surface of the shell. We use $\overline{\mathbf{x}}: \omega \rightarrow \bar{\Omega}$ and $\mathbf{x}: \omega \rightarrow \Omega_{t}$ to denote the mappings from the mid-surface parameter domain to the reference $(\bar{\Omega})$ and time $t\left(\Omega_{t}\right)$ configurations of the mid-surface. Similarly we use $\overline{\mathbf{r}}: \omega^{\tau} \rightarrow \bar{\Omega}^{\tau}$ and $\mathbf{r}: \omega^{\tau} \rightarrow \Omega_{t}^{\tau}$ to denote mappings from the shell parameter domain to the reference $\left(\bar{\Omega}^{\tau}\right)$ and time $t\left(\Omega_{t}^{\tau}\right)$ configurations of the shell. We illustrate this in Figure 6 . We will use $\xi=\left(\xi_{1}, \xi_{2}, \xi_{3}\right) \in \omega^{\tau}$ to denote coordinates in parameter space. We refer to surfaces $\mathbf{s}\left(\xi_{1}, \xi_{2}\right)=$ $\mathbf{r}\left(\xi_{1}, \xi_{2}, \hat{\xi}_{3}\right)$ in the shell with fixed values of the thickness parameter $\hat{\xi}_{3}$ as laminae and we refer to lines in the $\mathbf{l}\left(\xi_{3}\right)=\mathbf{r}\left(\hat{\xi}_{1}, \hat{\xi}_{2}, \xi_{3}\right)$ with fixed values of the surface parameters $\hat{\xi}_{1}, \hat{\xi}_{2}$ as fibers. We illustrate fibers and laminae in Figure 7.

\section{SHELL KINEMATICS}

We assume the kinematics of a continuum shell

$$
\overline{\mathbf{r}}(\xi)=\overline{\mathbf{x}}\left(\xi_{1}, \xi_{2}\right)+\xi_{3} \overline{\mathbf{a}}_{3}\left(\xi_{1}, \xi_{2}\right), \mathbf{r}(\xi)=\mathbf{x}\left(\xi_{1}, \xi_{2}\right)+\xi_{3} \mathbf{a}_{3}\left(\xi_{1}, \xi_{2}\right)
$$

where $\overline{\mathbf{a}}_{3}$ is the unit normal to the mid-surface and $\mathbf{a}_{3}$ is the stretched and sheared image of $\overline{\mathbf{a}}_{3}$ under the motion of the shell. We use $\overline{\mathbf{a}}_{\alpha}=\frac{\partial \overline{\mathrm{x}}}{\partial \xi_{\alpha}}$ to denote the tangents to the mid-surface of the reference shell. When combined with $\overline{\mathbf{a}}_{3}=\frac{\overline{\mathbf{a}}_{1} \times \overline{\mathbf{a}}_{2}}{\left|\overline{\mathbf{a}}_{1} \times \overline{\mathbf{a}}_{2}\right|}$, they form a complete basis for $\mathbb{R}^{3}$ (see Figure 7).

We decompose the motion of the shell into two steps

$$
\mathbf{r}(\xi)=\phi^{S}\left(\mathbf{r}^{K L}(\xi)\right)
$$

The first step $\mathbf{r}^{K L}: \omega^{\tau} \rightarrow \Omega_{t}^{K L, \tau}$ does not see shearing or compression normal to the mid-surface. That is, lines originally normal to the midsurface rotate and translate with the midsurface so that 

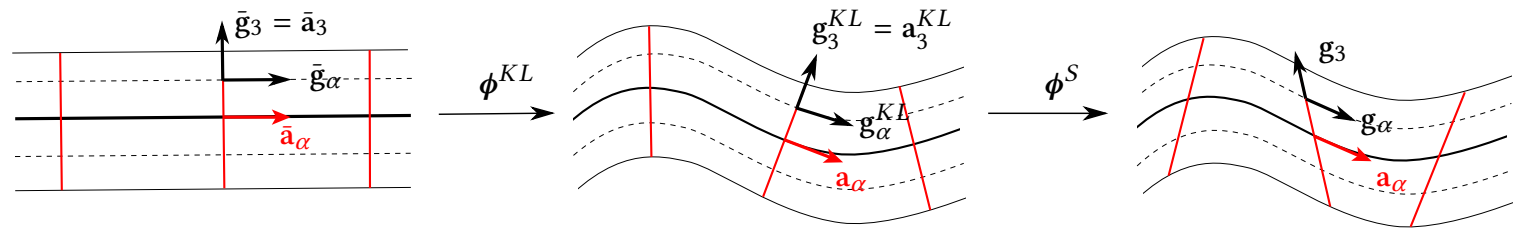

Fig. 7. Continuum shell/Kirchhoff-Love splitting. Mid-surface tangents and fibers are shown in red. Laminae are shown as dashed curves, and the local frame at a point on a lamina is shown in black. On the left is the undeformed reference configuration, while the deformed configuration is on the right, and the middle shows the intermediate Kirchhoff-Love deformation.

they remain constant length and normal to the midsurface. This is consistent with a Kirchhoff-Love kinematic assumption

$$
\mathbf{r}^{K L}(\xi)=\mathbf{x}\left(\xi_{1}, \xi_{2}\right)+\xi_{3} \mathbf{a}_{3}^{K L}\left(\xi_{1}, \xi_{2}\right)
$$

Here $\mathbf{a}_{3}^{K L}$ is the unit normal to the mid-surface which satifsies $\mathbf{a}_{3}^{K L}=\frac{\mathbf{a}_{1} \times \mathbf{a}_{2}}{\left|\mathbf{a}_{1} \times \mathbf{a}_{2}\right|}$ where $\mathbf{a}_{\alpha}=\frac{\partial \mathbf{x}}{\partial \xi_{\alpha}}$. The second step $\phi^{S}: \Omega_{t}^{K L, \tau} \rightarrow$ $\Omega_{t}^{\tau}$ does not move the mid-surface but captures the shearing and compression/extension of material normal to the mid-surface. That is, lines that remained normal to the midsurface and with constant length in the Kirchhoff-Love mapping $\mathbf{r}^{K L}$ are allowed to change length and shear under the mapping $\phi^{S}$, thus becoming non-normal to the midsurface in general (see Figure 7).

\subsection{Deformation Gradient}

The motion of the shell from the reference configuration to the time $t$ configuration is then obtained from the composition $\phi$ $\bar{\Omega}^{\tau} \rightarrow \Omega_{t}^{\tau}, \boldsymbol{\phi}(\mathrm{X})=\mathbf{r}\left(\overline{\mathbf{r}}^{-1}(\mathrm{X})\right)$ for $\mathrm{X} \in \bar{\Omega}^{\tau}$. The elastic and frictional contact responses of our model are characterized in terms of the spatial derivative (our deformation gradient) of this mapping. The deformation gradient of the motion is $\mathrm{F}=\frac{\partial \phi}{\partial \mathrm{X}}=\frac{\partial \mathrm{r}}{\partial \xi}\left(\frac{\partial \overline{\mathrm{r}}}{\partial \xi}\right)^{-1}$, which can further be expressed in terms of derivatives from the parameter space $\mathbf{g}_{i}=\frac{\partial \mathrm{r}}{\partial \xi_{i}}$ and $\overline{\mathbf{g}}_{i}=\frac{\partial \overline{\mathbf{r}}}{\partial \xi_{i}}$ as $\mathbf{F}=\mathbf{g}_{i} \otimes \overline{\mathbf{g}}^{i}$. Here $\overline{\mathbf{g}}^{i}$ are the contravariant basis vectors associated with $\overline{\mathbf{g}}_{i}$. Furthermore, the composition of motion in Equation (2) leads to the multiplicative decomposition

$$
\mathbf{F}=\mathbf{F}^{S} \mathbf{F}^{K L}, \quad \mathbf{F}^{S}=\mathbf{g}_{i} \otimes \mathbf{g}^{K L, i}, \quad \mathbf{F}^{K L}=\mathbf{g}_{i}^{K L} \otimes \overline{\mathbf{g}}^{i}
$$

where $\mathbf{g}_{i}^{K L}=\frac{\partial \mathbf{r}^{K L}}{\partial \xi_{i}}$ and $\mathbf{g}^{K L, j}$ form the corresponding contravariant basis. We note that the third contravariant counterparts to the Kirchhoff-Love and material configuration bases are the same as their covariant counterparts because of the perservation of midsurface normals in these mappings. That is, $\mathrm{g}_{3}^{K L}=\mathrm{g}^{K L, 3}=\mathbf{a}_{3}^{K L}$ and $\overline{\mathbf{g}}_{3}=\overline{\mathbf{g}}^{3}=\overline{\mathbf{a}}_{3}$ since $\mathbf{g}_{\alpha}^{K L} \cdot \mathbf{g}_{3}^{K L}=0$ and $\overline{\mathbf{g}}_{\alpha} \cdot \overline{\mathbf{g}}_{3}=0$ (see [Clyde 2017] for details).

\subsection{Plasticity}

As in Jiang et al.[2017], we use an elastoplastic decomposition of the motion to resolve frictional contact. Following that approach, we allow for plastic deformation in the fiber directions to enable material separation and frictional sliding. However, in order to decouple the frictional contact stress from the bending stress, we only apply the frictional contact elastoplastic decomposition to the shearing component of the motion. Furthermore, unlike in Jiang et al.[2017] we also allow for plastic deformation in the laminae to account for yielding and denting of the shell. This plastic decomposition is applied to the motion in the Kirchhoff-Love component of the motion.

The frictional contact elastic stress model in Jiang et al. [2017] penalizes compression and shearing of the surface normals. Since the Kirchhoff-Love component of the motion does not see any sliding or compression relative to the mid-surface, it is not capable of resolving frictional contact in this manner. We therefore apply this model to the shearing and compression/extension component of the shearing motion $\mathbf{F}^{S}=\mathbf{F}^{S, E} \mathbf{F}^{S, P}$ as

$$
\begin{aligned}
& \mathbf{F}^{S, E}=\mathbf{g}_{\alpha} \otimes \mathbf{g}^{K L, \alpha}+\mathbf{a}_{3}^{E} \otimes \mathbf{g}_{3}^{K L}, \\
& \mathbf{F}^{S, P}=\mathbf{g}_{\alpha}^{K L} \otimes \mathbf{g}^{K L, \alpha}+\mathbf{a}_{3}^{P} \otimes \mathbf{g}_{3}^{K L} .
\end{aligned}
$$

Here $\mathbf{a}_{3}^{E}$ represents the shearing and compression/extension of normals in the shell that is penalized elastically. Coulomb friction constrains how much shearing and compression is penalized. $\mathbf{a}_{3}^{P}$ is the discarded shearing and extension in the fiber direction from plastic yielding associated with this constraint. They are related through $\mathbf{F}^{S, E} \mathbf{a}_{3}^{P}=\mathbf{a}_{3}$. We note $\mathbf{F}^{S, P}$ does not affect components in the laminae since we do not want the frictional contact response to couple with the elastoplasticty of the Kirchhoff-Love component of the shell motion.

To allow for yielding and denting of the shell in response to loading, we decompose the Kirchhoff-Love component of the motion into lamina elastic and plastic parts $\mathrm{F}^{K L}=\mathrm{F}^{K L, E} \mathrm{~F}^{K L, P}$

$$
\begin{aligned}
& \mathbf{F}^{K L, E}=\mathbf{g}_{\alpha}^{K L} \otimes \mathbf{g}^{P, \alpha}+\mathbf{g}_{3}^{K L} \otimes \overline{\mathbf{g}}_{3}, \\
& \mathbf{F}^{K L, P}=\mathbf{g}_{\alpha}^{P} \otimes \overline{\mathbf{g}}^{\alpha}+\overline{\mathbf{g}}_{3} \otimes \overline{\mathbf{g}}_{3}
\end{aligned}
$$

Here the form of $\mathrm{F}^{K L, P}$ is designed to not affect the motion normal to the mid-surface since the elastoplasiticty of denting and wrinkling is expressed only in terms of the lamina components of defomraiton. The exprssion for $\mathrm{F}^{K L, E}$ is then what remains to satisfy the constraint $\mathbf{F}^{K L}=\mathbf{F}^{K L, E} \mathbf{F}^{K L, P}$. We note that the $\mathbf{g}_{\alpha}^{P}$ (with $\mathrm{g}_{\alpha}^{P} \cdot \overline{\mathbf{g}}_{3}=0$ ) in Equation (8) for $\mathrm{F}^{K L, P}$ express the forgotten deformation of plastic yielding in the lamina that is associated with denting and wrinkling. The $\left\{\mathrm{g}^{P \alpha}, \overline{\mathbf{g}}_{3}\right\}$ are the contravariant counterparts to $\left\{\mathbf{g}_{\alpha}^{P}, \overline{\mathbf{g}}_{3}\right\}$. Lastly, $\overline{\mathbf{g}}_{3}$ is the same in the covariant and contravariant bases as in Equation (4). 


\section{ELASTIC STRESS AND PLASTIC CONSTRAINTS}

We define our elastoplastic constitutive response to deformation and frictional contact terms of potential energy in the shell. We decompose the total elastic potential as a sum of contributions from the Kirchhoff-Love (lamina elasticity, denting wrinkling etc.) and shearing (frictional contact) potentials. The contribution from the Kirchhoff-Love motion is

$$
\Psi^{K L}=\int_{\omega} \int_{-\frac{\tau}{2}}^{\frac{\tau}{2}} \psi\left(\mathbf{F}^{K L, E}\right)\left|\frac{\partial \overline{\mathbf{r}}}{\partial \xi}\right| d \xi .
$$

and the total elastic potential energy of the shell is

$$
\Psi^{C S}=\Psi^{K L}+\int_{\omega} \int_{-\frac{\tau}{2}}^{\frac{\tau}{2}} \chi\left(\mathbf{F}^{S, E}\right)\left|\frac{\partial \overline{\mathbf{r}}}{\partial \xi}\right| d \xi
$$

where $\psi\left(\mathbf{F}^{K L, E}\right)$ is the elastic potential energy density of the KirchhoffLove motion and $\chi\left(\mathrm{F}^{S, E}\right)$ is the energy density of the normal shearing and compression in the continuum shell motion.

These potentials are defined from energy densities $\psi\left(\mathbf{F}^{K L, E}\right)$ and $\chi\left(\mathrm{F}^{S, E}\right)$ respectively. In general, a potential energy density $\Xi$ of this type is related to the material Kirchhoff stress $\tau$ through $\tau=\frac{\partial \Xi\left(\mathrm{F}^{E}\right)}{\partial \mathrm{F}^{E}} \mathbf{F}^{E}$. It is the stress defined by this relationship that will directly affect our MPM implementation. In our elastoplastic model, the stress must satisfy certain constraints related to bending and denting as well as frictional contact. In the sections that follow we define these elastic stresses and their associated plastic constraints.

\section{BENDING AND LAMINA POTENTIAL}

The energy density $\psi\left(\mathbf{F}^{K L, E}\right)$ penalizes only the deformation in the laminae (zero transverse normal stress) since the Kirchhoff-Love kinematics preclude shearing and compression of the fibers. The stress in the material is the derivative of this potential with respect to strain (see [Guo et al. 2018] for derivation). Our approach supports any potential used in Kirchhoff-Love shell models. In particular we use the orthotropic model for woven fabrics from Clyde et al.[2017] in Figures 13a and 13b. Here we provide the derivation of a simple energy density useful for applications with denting that is isotropic in the lamina directions while satisfying the zero transverse normal stress condition.

With Kirchhoff-Love kinematics, the lamina directions $\overline{\mathbf{g}}_{\alpha}=$ $\overline{\mathbf{a}}_{\alpha}+\xi_{3} \overline{\mathbf{a}}_{3, \alpha}$ and $\mathbf{g}_{\alpha}^{K L}=\mathbf{a}_{\alpha}+\xi_{3} \mathbf{a}_{3, \alpha}^{K L}$ are always tangent to the midsurface since $\overline{\mathbf{g}}_{\alpha} \cdot \overline{\mathbf{a}}_{3}=\mathbf{g}_{\alpha}^{K L} \cdot \mathbf{a}_{3}^{K L}=0$. In order to satisfy the zero transverse normal stress conditions, we design a potential density with respect to the lamina directions by first writing the KirchhoffLove deformation in the reference mid-surface lamina/fiber basis $\mathbf{F}^{K L, E}=F_{i j}^{K L, E} \overline{\mathbf{a}}_{i} \otimes \overline{\mathbf{a}}_{j}$. Here the directions $\overline{\mathbf{a}}_{\alpha}$ are the tangents to the midsurface in the reference configuration and $\overline{\mathbf{a}}_{3}$ is the normal. This choice of basis more clearly identifies deformations in the laminae and normal directions since $F_{\alpha \beta}^{K L, E}$ are then components of deformation in the laminae. The right Cauchy-Green strain is $\mathbf{C}=C_{i j} \overline{\mathbf{a}}_{i} \otimes \overline{\mathbf{a}}_{j}$

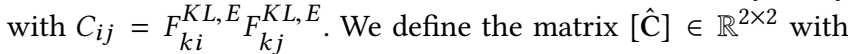
entries $C_{\alpha \beta}$. This is the upper left $2 \times 2$ block of the matrix of $C_{i j}$ entries and it represents strain in the lamina. We use a model that is quadratic in the right Hencky strain $\left[\epsilon^{R}\right]=\frac{1}{2} \log ([\hat{\mathbf{C}}])$

$$
\psi\left(\mathbf{F}^{K L, E}\right)=\mu \epsilon_{\alpha \beta}^{R} \epsilon_{\alpha \beta}^{R}+\frac{\lambda}{2}\left(\epsilon_{\delta \delta}^{R}\right)^{2} .
$$

Here the $\epsilon_{\alpha \beta}^{R}$ are the entries in $\left[\epsilon^{R}\right] \in \mathbb{R}^{2 \times 2}$ and $\mu, \lambda$ are Lame parameters that can be set intuitively from Young's modulus and Poisson ratio to control stiffness and incompressibility in the lamina. We choose the quadratic in Hencky strain model because it simplifies the return mapping during plastic yielding (see Section §7.1).

It is convenient for our MPM implementation as well as for the plasticity constraints to work with the Kirchhoff stress $\tau$. It is related to the more commonly used Cauchy stress $\sigma$ as $\tau=\operatorname{det}(F) \sigma$. The derivation of the Kirchhoff stress in terms of the potential is given in the the supplementary technical document [Guo et al. 2018]. We summarize the expression as

$$
\tau^{K L}=\tau_{\alpha \beta} \mathbf{q}_{\alpha}^{K L, E} \otimes \mathbf{q}_{\beta}^{K L, E}, \quad \tau_{\alpha \beta}^{K L}=2 \mu \epsilon_{\alpha \beta}^{L}+\lambda \epsilon_{\gamma \gamma}^{L} \delta_{\alpha \beta} .
$$

Here we write the stress in terms of the basis defined by the directions $\mathbf{q}_{i}^{K L, E}$ obtained from the QR decomposition $\mathbf{F}^{K L, E}=r_{i j}^{K L, E} \mathbf{q}_{i}^{K L, E} \otimes$ $\overline{\mathbf{a}}_{j}$ with respect to the reference lamina/fiber basis $\overline{\mathbf{a}}_{j}$. Since the Kirchhoff-Love component of the motion preserves normals to the midsurface, the first two directions $\mathbf{q}_{\alpha}^{K L, E}$ are tangent to the deformed lamina and the third direction $\mathrm{q}_{3}^{K L, E}$ is normal to the midsurface. Therefore, since $\tau^{K L}$ is expressed only in terms of $\mathbf{q}_{\alpha}^{K L, E}$, we see that it satisfies the zero transverse normal stress condition since it has no components in the directions normal to the laminae. We use $\epsilon_{\alpha \beta}^{L}$ to denote the entries in the left Hencky strain matrix $\left[\boldsymbol{\epsilon}^{L}\right]=\frac{1}{2} \log \left(\left[\hat{\mathbf{r}}^{K L, E}\right]\left[\hat{\mathbf{r}}^{K L, E}\right]^{T}\right) \in \mathbb{R}^{2 \times 2}$. Here, $\left[\hat{\mathbf{r}}^{K L, E}\right] \in \mathbb{R}^{2 \times 2}$ is the matrix with entries $r_{\alpha \beta}^{K L, E}$. These are the components of the deformation gradient $\mathrm{F}^{K L, E}$ related to the lamina strain. This formula follows directly from the definition of the energy in Equation (11) and we provide details of the derivation in the supplementary technical document [Guo et al. 2018].

\subsection{Denting Yield Condition and Return Mapping}

In order to produce permanent denting and wrinkling phenomena resulting from excessive straining, we introduce a notion of yield stress. Intuitively, stresses satisfying the yield stress criteria are those associated with elastic, non-permanent deformation in the shell. Those that do not satisfy the condition are non-physical and permanent plastic deformation will occur to prevent them from happening. We apply the von Mises yield condition to the KirchhoffStress in Equation (12). This condition states that the shear stress (or magnitude of the deviatoric component of the stress) must be less than a threshold $c_{v M}$ before permanent plastic deformation occurs

$$
f_{v M}(\boldsymbol{\tau})=\left|\boldsymbol{\tau}-\frac{\operatorname{tr}(\boldsymbol{\tau})}{3} \mathbf{I}\right|_{F} \leq c_{v M}
$$

This condition defines a cylindrical region of feasible states in the principal stress space since

$$
f_{v M}(\tau)=\sqrt{\frac{2}{3}\left(\tau_{1}^{2}+\tau_{2}^{2}+\tau_{3}^{2}-\left(\tau_{1} \tau_{2}+\tau_{2} \tau_{3}+\tau_{1} \tau_{3}\right)\right)}
$$

where $\boldsymbol{\tau}=\sum_{i} \tau_{i} \mathbf{u}_{i} \otimes \mathbf{u}_{i}$ with principal stresses $\tau_{i}$. Stresses with principal values in the cylinder do not produce any permanent 
deformation. Note that zero stress is inside the cylinder. As deformation becomes significant enough that the principal stresses reach the boundary of the cylinder, permanent plastic denting and wrinkling will occur. The zero transverse normal stress nature of $\tau^{K L}=\sum_{\alpha} \tau_{\alpha}^{K L} \mathbf{u}_{\alpha} \otimes \mathbf{u}_{\alpha}$ means that its principal stresses are always in a plane and thus the feasible region is ellipsoidal intersection of the cylinder and the plane (see supplementary technical document [Guo et al. 2018] for illustration).

In practice, the yield condition is satisfied via projection (or return mapping) of the stress to the feasible region. During simulation, we first take a time step to create a trial state of stress ignoring the yield condition. By ignoring the condition, we essentially assume the material undergoes no further plastic deformation. We use $\mathbf{F}^{K L, E^{\operatorname{tr}}}$, $\mathrm{F}^{K L, P^{\mathrm{tr}}}$ to denote this trial state of elastoplastic strains with associated trial stress $\tau^{K L^{\operatorname{tr}}}$. This stress may or may not satisfy the yield condition. The trial stress $\tau^{K L^{\text {tr }}}$ is then projected to the feasible region to create $\tau^{K L}$ which satisfies the yield condition. The elastic and plastic strains are then computed from the projected stress. We use $\mathbf{F}^{K L, E}, \mathrm{~F}^{K L, P}$ to denote final elastic and plastic deformation associated with the projected stress $\tau^{K L}$. The product of the projected elastic and plastic deformation gradients must be equal to the original deformation gradient, creating a constraint on the return mapping

$$
\mathbf{F}^{K L}=\mathbf{F}^{K L, E^{\operatorname{tr}}} \mathbf{F}^{K L, P^{\operatorname{tr}}}=\mathbf{F}^{K L, E} \mathbf{F}^{K L, P} .
$$

We describe the process as $\mathbf{F}^{K L, E^{\operatorname{tr}}}, \mathbf{F}^{K L, P^{\operatorname{tr}}} \rightarrow \mathrm{F}^{K L, E}, \mathbf{F}^{K L, P}$.

The projection is naturally done in terms of the $\mathrm{QR}$ decomposition of the trial elastic deformation gradient $\mathbf{F}^{K L, E^{\operatorname{tr}}}=r_{\alpha \beta}^{K L, E^{\operatorname{tr}}} \mathbf{q}_{\alpha}^{K L, E} \otimes$ $\overline{\mathbf{a}}_{\beta}+\mathbf{q}_{3}^{K L, E} \otimes \overline{\mathbf{a}}_{3}$. The trial principle stresses are

$$
\begin{aligned}
& \tau_{1}^{K L, \operatorname{tr}}=(2 \mu+\lambda) \log \left(\sigma_{1}^{E \operatorname{tr}}\right)+\lambda \log \left(\sigma_{2}^{E \operatorname{tr}}\right) \\
& \tau_{2}^{K L, \operatorname{tr}}=(2 \mu+\lambda) \log \left(\sigma_{2}^{E \operatorname{tr}}\right)+\lambda \log \left(\sigma_{1}^{E \operatorname{tr}}\right)
\end{aligned}
$$

where $\sigma_{\alpha}^{E \operatorname{tr}}$ are the singular values of the matrix $\left[\hat{\mathbf{r}}^{K L, E \operatorname{tr}}\right] \in \mathbb{R}^{2 \times 2}$ with entries $r_{\alpha \beta}^{K L, E \operatorname{tr}}$ from the QR decomposition

$$
\left[\hat{\mathbf{r}}^{K L, E \operatorname{tr}}\right]=\left[\mathbf{U}^{E}\right]\left(\begin{array}{ll}
\sigma_{1}^{E^{\operatorname{tr}}} & \\
& \sigma_{2}^{E^{\operatorname{tr}}}
\end{array}\right)\left[\mathbf{V}^{E}\right]^{T} .
$$

We project the trial $\tau_{\alpha}^{K L \text {,tr }}$ to the intersection of the von Mises yield surface and the $(1,2)$ plane to obtain the projected $\tau_{\alpha}^{K L}$ from which

$$
\left(\begin{array}{c}
\log \left(\sigma_{1}^{E}\right) \\
\log \left(\sigma_{2}^{E}\right)
\end{array}\right)=\left(\begin{array}{cc}
2 \mu+\lambda & \lambda \\
\lambda & 2 \mu+\lambda
\end{array}\right)^{-1}\left(\begin{array}{c}
\tau^{K L}{ }_{1} \\
\tau^{K L_{2}}
\end{array}\right) .
$$

We then express the deformation gradient associated with this stress projection as $\mathbf{F}^{K L, E}=F_{\alpha \beta}^{K L, E} \mathbf{q}_{\alpha}^{K L, E} \otimes \overline{\mathbf{a}}_{\beta}+\mathbf{q}_{3}^{K L, E} \otimes \overline{\mathbf{a}}_{3}$ where $F_{\alpha \beta}^{K L, E}$ are the components of the elastic deformation gradient

$$
\left[\hat{\mathbf{F}}^{K L, E}\right]=\left[\mathbf{U}^{E}\right]\left(\begin{array}{cc}
\sigma_{1}^{E} & \\
& \sigma_{2}^{E}
\end{array}\right)\left[\mathbf{V}^{E}\right]^{T} .
$$

The projected plastic deformation gradient is computed from $\mathrm{F}^{K L, P}=$ $\mathbf{F}^{K L, E^{-1}} \mathbf{F}^{K L}$ in order to maintain the constraint in Equation (15). We provide more detail in this derivation in the supplementary technical document [Guo et al. 2018].

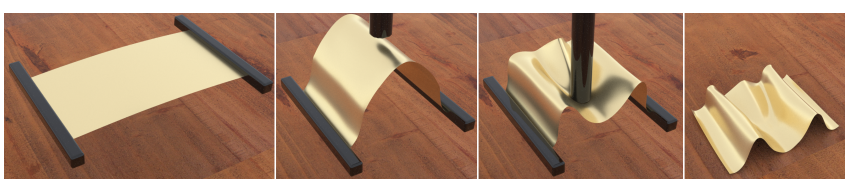

Fig. 8. Denting. We demonstrate plastic deformation of foil induced by object collision.

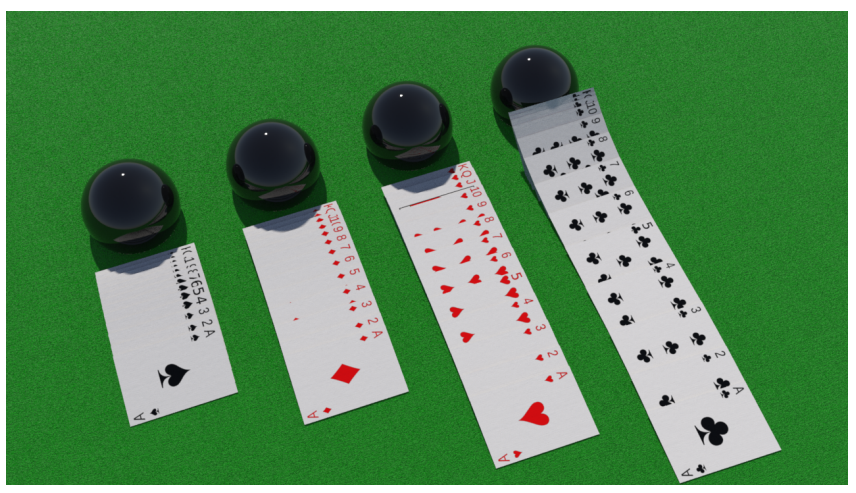

Fig. 9. Variation in Coulomb friction coefficient. The effect of the friction parameter $c_{F}$ can be seen in this card comparison. By decreasing $c_{F}$ (from left to right) we demonstrate a range of surface frictions.

7.1.1 Associativity and Hencky Strain. The projection of the trial stress to the feasible region is done using a generalized notion of closest point. This generalized projection is derived from the associative plastic flow assumption. Associativity requires that the closest points to the feasible stress region are not traced back along lines normal to the boundary, but rather along lines parallel to a matrix times the normal [Bonet and Wood 2008]. This matrix is associated with the linearization of the constitutive model and in general it varies along the boundary. However, with the quadratic in Hencky strain model given in Equation (11), the matrix is constant along the boundary of the feasible region, which greatly simplifies the process of finding the generalized closest point. We illustrate this further in the supplementary technical document [Guo et al. 2018].

\section{FRICTIONAL CONTACT POTENTIAL}

As in Jiang et al.[2017], we resolve collision and contact through the continuum. We design the potential energy density $\chi\left(\mathrm{F}^{S, E}\right)$ to penalize compression and shearing in the direction normal to the mid-surface as in Jiang et al.[2017]. The deformation of the fiber from the Kirchhoff-Love configuration is given by $\mathbf{a}_{3}^{E}=\mathbf{F}^{S, E} \mathbf{a}_{3}^{K L}$. We decompose this into shear $\left(\mathbf{a}_{3 S}^{E}\right)$ and normal $\left(s_{3}^{E} \mathbf{a}_{3}^{K L}\right)$ components $\mathbf{a}_{3}^{E}=\mathbf{a}_{3 S}^{E}+s_{3}^{E} \mathbf{a}_{3}^{K L}$ where $s_{3}^{E}=\mathbf{a}_{3}^{E} \cdot \mathbf{a}_{3}^{K L}$. As material normal to the cloth is compressed, the normal component $s_{3}^{E}$ will decrease and as the material separates, it will increase. Similarly, as material slides tangentially to the shell $\left|\mathbf{a}_{3 S}^{E}\right|$ will increase. We therefore write our potential as

$$
\chi\left(\mathbf{F}^{S, E}\right)=\frac{\gamma}{2}\left|\mathbf{a}_{3 S}^{E}\right|^{2}+f\left(s_{3}^{E}\right)
$$


where $\gamma$ represents the amount of shear resistance and

$$
f\left(s_{3}^{E}\right)=\left\{\begin{array}{cc}
\frac{k^{c}}{3}\left(1-s_{3}^{E}\right)^{3} & 0 \leq s_{3}^{E} \leq 1 \\
0 & s_{3}^{E}>1
\end{array}\right.
$$

represents the resistance to compression/contact which increases with the parameter $k^{c}>0$. This potential is designed to increase, and thus penalize, increasing compressive contact and shear. Note that in the case of fiber elongation $\left(s_{3}^{E}>1\right)$ there is no elastic penalty as this would be associated with cohesive contact.

The potential in Equation (21) is constant in the fiber direction since $\mathbf{a}_{3}^{K L}$ is constant along the fiber from the continuum shell kinematics. Therefore it is convenient to express the contact potential $\chi$ at all points in the fibers in terms of their values at the mid-surface $\chi\left(\mathbf{F}^{S, E}\left(\xi_{1}, \xi_{2}, \xi_{3}\right)\right)=\chi\left(\mathbf{F}^{S, E}\left(\xi_{1}, \xi_{2}, 0\right)\right)$ since

$$
\int_{\omega} \int_{-\frac{\tau}{2}}^{\frac{\tau}{2}} \chi\left(\mathbf{F}^{S, E}\right)\left|\frac{\partial \overline{\mathbf{r}}}{\partial \xi}\right| d \xi=\int_{\omega} \chi\left(\mathbf{F}^{S, E}\right) \int_{-\frac{\tau}{2}}^{\frac{\tau}{2}}\left|\frac{\partial \overline{\mathbf{r}}}{\partial \xi}\right| d \xi
$$

in Equation (10). On the mid-surface $\mathbf{F}^{S, E}\left(\xi_{1}, \xi_{2}, 0\right)=\mathbf{a}_{\alpha} \otimes \mathbf{a}^{K L, \alpha}+$ $\mathbf{a}_{3}^{E} \otimes \mathbf{a}_{3}^{K L}$. Furthermore, since the potential varies with the normal and tangential components of $\mathbf{a}_{3}^{E}$, it is equivalent to write the energy as a function of the tensor $\mathbf{a}_{\alpha} \otimes \overline{\mathbf{a}}_{\alpha}+\mathbf{a}_{3}^{E} \otimes \overline{\mathbf{a}}_{3}$ since its $\mathrm{QR}$ decomposition with respect to the $\overline{\mathbf{a}}_{i}$ basis satisfies

$$
\mathbf{a}_{\alpha} \otimes \overline{\mathbf{a}}_{\alpha}+\mathbf{a}_{3}^{E} \otimes \overline{\mathbf{a}}_{3}=r_{i j}^{S, E} \mathbf{q}_{i}^{S, E} \otimes \overline{\mathbf{a}}_{j}
$$

and the energy density can then be written in terms of the QR decomposition as was done in Jiang et al.[2017]

$$
\chi\left(\mathbf{F}^{S, E}\left(\xi_{1}, \xi_{2}, 0\right)\right)=\frac{\gamma}{2}\left(r_{13}^{S, E^{2}}+r_{23}^{S, E^{2}}\right)+f\left(r_{33}^{S, E}\right) .
$$

This follows because the normal and shear components of $\mathbf{a}_{3}^{E}$ can be written in terms of the basis vectors $\mathrm{q}_{i}^{S, E}$ from the QR decomposition $\mathbf{a}_{3}^{E}=r_{i 3}^{S, E} \mathbf{q}_{i}^{S, E}$. With this convention, $s_{3}^{E}=r_{33}^{S, E} \operatorname{since} \operatorname{span}\left\{\mathbf{a}_{\alpha}\right\}=$ $\operatorname{span}\left\{\mathbf{q}_{\alpha}^{S, E}\right\}$ and $\mathbf{q}_{3}^{S, E}=\mathbf{a}_{3}^{K L}$. Using $s_{i}^{E}=r_{i 3}^{S, E}$ for conciseness

$$
\boldsymbol{\tau}^{S}=\gamma s_{i}^{E} s_{j}^{E} \mathbf{q}_{i}^{S, E} \otimes \mathbf{q}_{j}^{S, E}+\left(f^{\prime}\left(s_{3}^{E}\right) s_{3}^{E}-\gamma s_{3}^{E^{2}}\right) \mathbf{q}_{3}^{S, E} \otimes \mathbf{q}_{3}^{S, E} .
$$

We provide a more detailed derivation of energies defined in terms of the $\mathrm{QR}$ decomposition and this specific case in the supplementary technical document [Guo et al. 2018].

\subsection{Frictional Contact Yield Condition and Return Mapping}

With a continuum view of frictional contact, Coulomb friction defines a constraint on the types of stress that are admissible. This can be done concisely in terms of the Cauchy stress $\sigma$. This stress measure is defined through contact interactions internal to a continuum body [Gonzalez and Stuart 2008]. Specifically, the contact force per unit area across a surface with normal $\mathbf{n}$ is $\sigma \mathbf{n}$. In the shell, the contact direction is $\mathbf{a}_{3}^{K L}$. Coulomb friction places a constraint on the stress as

$$
\left|\mathbf{t}_{S}\right| \leq-c_{F} \sigma_{n}
$$

where $\sigma \mathbf{a}_{3}^{K L}=\sigma_{n} \mathbf{a}_{3}^{K L}+\mathbf{t}_{S}$. Here $\sigma \mathbf{a}_{3}^{K L}$ is contact force per unit area, $\sigma_{n} \mathbf{a}_{3}^{K L}$ is its normal component and $\mathbf{t}_{S}$ is the shearing component orthogonal to $\mathbf{a}_{3}^{K L}$. The condition in Equation (27) states that the magnitude of the shearing component can be no larger than a coefficient of friction times the normal component, with the convention that no shearing is allowed in the case of $\sigma_{n}>0$ since this would be a separating rather than a compressive state. We note that each object can have its own coefficient of friction which provides a simple way of modeling interactions between many objects.

The Kirchhoff stress is related to the Cauchy stress as $\tau=\operatorname{det}(\mathbf{F}) \sigma$. By design, the Kirchhoff-Love Kirchhoff stress has no component in the $\mathbf{a}_{3}^{K L}$ direction $\tau^{K L} \mathbf{a}_{3}^{K L}=\mathbf{0}$. Therefore, the Coulomb friction constraint applies only to the shearing Kirchhoff stress $\tau^{S}$. Using Equation (26) we can see that the continuum stress Coulomb friction condition is

$$
\sqrt{s_{1}^{E^{2}}+s_{2}^{E^{2}}} \leq\left\{\begin{array}{cc}
\frac{c_{F} k^{c}}{\gamma}\left(1-s_{3}^{E}\right)^{2}, & 0<s_{3}^{E} \leq 1 \\
0, & s_{3}^{E}>1
\end{array}\right.
$$

Whereas the plastic constraint associated with denting involved the principle stresses of $\tau^{K L}$, only the components $s_{i}^{E}$ of the elastic $\mathbf{a}_{3}^{E}$ in the $\mathrm{q}_{i}^{S, E}$ basis are constrained under the Coulomb condition. It is satisfied with a return mapping of trial elastic $\mathbf{a}_{3}^{E^{\operatorname{tr}}}=s_{i}^{E^{\operatorname{tr}}} \mathbf{q}_{i}^{S, E}$ to the projected $\mathbf{a}_{3}^{E}=s_{i}^{E} \mathbf{q}_{i}^{S, E}$ where the trial and projected coefficients are related through

$s_{\alpha}^{E}=\left\{\begin{array}{cc}h\left(\mathbf{a}_{3}^{E^{\operatorname{tr}}}\right) s_{\alpha}^{E^{\operatorname{tr}}}, & 0<s_{3}^{E^{\operatorname{tr}}} \leq 1 \\ 0, & s_{3}^{E^{\operatorname{tr}}}>1\end{array}, s_{3}^{E}=\left\{\begin{array}{cc}s_{3}^{E^{\operatorname{tr}},} & 0<s_{3}^{E^{\operatorname{tr}}} \leq 1 \\ 1, & s_{3}^{E^{\operatorname{tr}}}>1\end{array}\right.\right.$

with

$$
h\left(\mathbf{a}_{3}^{E \operatorname{tr}}\right)=\left\{\begin{array}{cl}
\frac{c_{F} k^{c}\left(1-s_{3}^{E^{\operatorname{tr}}}\right)^{2}}{\gamma \sqrt{s_{1}^{\operatorname{tr}^{2}}+s_{2}^{\operatorname{tr}^{2}}}}, & \sqrt{s_{1}^{E \operatorname{tr}^{2}}+s_{2}^{E \operatorname{tr}^{2}}}>\frac{c_{F} k^{c}}{\gamma}\left(1-s_{3}^{E \operatorname{tr}}\right)^{2} \\
1, & \sqrt{s_{1}^{E \operatorname{tr}^{2}}+s_{2}^{E \operatorname{tr}^{2}}} \leq \frac{c_{F} k^{c}}{\gamma}\left(1-s_{3}^{E \operatorname{tr}}\right)^{2} .
\end{array}\right.
$$

This is the projection from Jiang et al.[2017] where $0<s_{3}^{E^{\text {tr }}} \leq 1$ implies material is compressed from contact in the normal direction. In this case, the function $h$ regulates the amount of shearing allowed relative to compression from the Coulomb constraint. In the case $s_{3}^{E^{\text {tr }}}>1$, material is separating in the normal direction and thus no resistance to shearing or compression is allowed.

\section{SUBDIVISION AND B-SPLINE FEM}

The Kirchhoff-Love kinematics require higher regularity for midsurface interpolating functions in FEM calculations. This arises from the use of the normal $\mathbf{a}_{3}^{K L}$ in the definition of the kinematics in Equation (3) since the deformation gradient in the shell then depends on second order derivatives of the kinematics of the midsurface. Technically the requirement is $H^{2}$ regularity, meaning that the interpolating functions and all their derivatives of order less than or equal to two are square integrable over the midsurface. In practice, this means that the interpolating functions must also have continuous first derivatives ( $C^{1}$ continuous) over the midsurface. This is a challenging constraint on the interpolating functions. We represent the shell midsurfaces as Catmull-Clark subdivision surfaces since they posses the required regularity.

The Catmull-Clark subdivision scheme takes as input an arbitrary polygonal mesh and returns a subdivided, refined mesh. The input polygonal mesh is referred to as the control mesh, and the limiting 


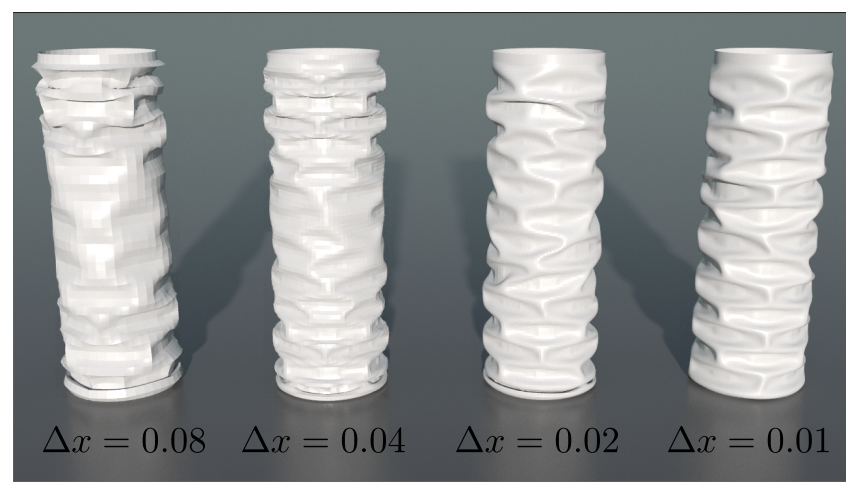

Fig. 10. Convergence under spatial refinement. We demonstrate that our method converges under refinement of grid and subd mesh spatial resolution in this buckling example. The simulations have increasing spatial resolution from left to right.

result of the subdivision process yields a $H^{2}$ surface [Catmull and Clark 1978; Stam 1998]. As the output mesh from Catmull-Clark subdivisions only consists of quadrilateral faces, we may assume that all input meshes have quadrilateral faces by replacing the control mesh with its first subdivision if necessary.

We denote the world space locations of the control points by $\mathbf{x}_{p}$, where $p=1, \ldots, n_{p}$ and $n_{p}$ is the number of control points. We use $\mathbf{x}^{K L}=\left(\mathbf{x}_{1}, \mathbf{x}_{2}, \ldots, \mathbf{x}_{n_{p}}\right)^{T}$ to denote the collection of all $\mathbf{x}_{p}$. The limiting surface from subdivision is represented as

$$
\mathbf{x}\left(\mathbf{x}^{K L}, \xi_{1}, \xi_{2}\right)=\mathbf{x}_{p} N_{p}^{S D}\left(\xi_{1}, \xi_{2}\right),
$$

where $N_{p}^{S D} \in H^{2}(\omega \rightarrow[0,1])$ is the FEM basis weight function corresponding to the control point $p$. The $N_{p}^{S D}$ have only local support and for each $\left(\xi_{1}, \xi_{2}\right) \in \omega$, only a sparse subset of $N_{p}^{S D}\left(\xi_{1}, \xi_{2}\right)$ are nonzero. We use the OpenSubdiv library to evaluate the basis functions $N_{p}^{S D}\left(\xi_{1}, \xi_{2}\right)$ and their first and second derivatives.

For each control mesh face, we sample rectangular quadrature points on either side of the face with $\xi_{3}=-\frac{\tau}{4}$ and $\xi_{3}=\frac{\tau}{4}$ for energy density evaluation. The generalized force on each of the control points is calculated as the negative derivative of the Kirchhoff-Love energy in Equation (10) which we approximate using quadrature

$$
\Psi^{K L}=\sum_{q} V_{q}^{0} \psi\left(\mathbf{F}_{q}^{K L, E \operatorname{tr}}\left(\mathbf{x}^{K L}\right)\right)
$$

The derivatives satisfy

$$
\begin{aligned}
\mathbf{f}_{p}^{K L} & =-\frac{\partial \Psi^{K L}\left(\mathbf{F}_{q}^{K L, E \operatorname{tr}}\left(\mathbf{x}^{K L}\right)\right)}{\partial \mathbf{x}_{p}} \\
& \left.=-\sum_{q} V_{q}^{0} \frac{\partial \psi}{\partial \mathbf{F}}\left(\mathbf{F}_{q}^{K L, E \operatorname{tr}}\left(\mathbf{x}^{K L}\right)\right)\right): \frac{\partial \mathbf{F}_{q}^{K L, E \operatorname{tr}}}{\partial \mathbf{x}_{p}}\left(\mathbf{x}^{K L}\right) .
\end{aligned}
$$

Here $\xi_{q 1}, \xi_{q 2}$ are the locations of the quadrature points in parameter space and $V_{q}^{0}$ are their associated volumes. For each quadrature point $q$, the Kirchhoff-Love deformation gradient at mid-surface

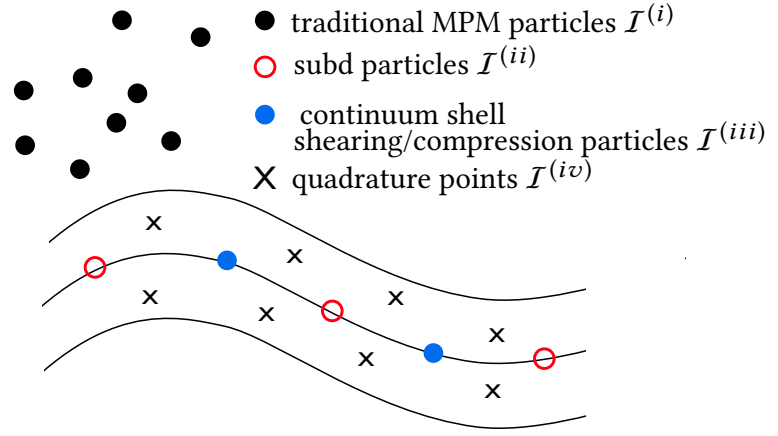

Fig. 11. Particle type classification. A schematic illustration of the different types of MPM particles and quadrature points.

configuration $\mathbf{x}^{K L}$ is computed from

$$
\mathrm{F}_{q}^{K L}\left(\mathrm{x}^{K L}\right)=\sum_{i=1}^{3} \mathrm{~g}_{q i}\left(\mathrm{x}^{K L}\right) \otimes \overline{\mathrm{g}}_{q}^{i} .
$$

Furthermore, in Equation (33),

$$
\frac{\partial \psi}{\partial \mathbf{F}}\left(\mathbf{F}_{q}^{K L, E \operatorname{tr}}\left(\mathbf{x}^{K L}\right)\right)=\tau^{K L}\left(\mathbf{F}_{q}^{K L, E \operatorname{tr}}\left(\mathbf{x}^{K L}\right)\right)\left(\mathbf{F}_{q}^{K L, E \operatorname{tr}}\left(\mathbf{x}^{K L}\right)\right)^{T}
$$

where $\tau^{K L}$ is from Equation (12). This relation follows from the definition of the first Piola-Kirchhoff stress and its relation to the Kirchhoff stress [Bonet and Wood 2008].

The trial elastic deformation $\mathrm{F}^{K L, E \operatorname{tr}}$ and its derivative with respect to control points $\frac{\partial \mathrm{F}_{q}^{K L, E \operatorname{tr}}}{\partial \mathrm{x}_{p}}\left(\mathrm{x}^{K L}\right)$ are computed assuming no further plastic flow over the time step

$$
\begin{aligned}
\mathbf{F}_{q}^{K L, E t r} & =\mathbf{F}_{q}^{K L} \mathbf{F}_{q}^{K L, P, n^{-1}} \\
\frac{\partial \mathbf{F}_{q}^{K L, E \operatorname{tr}}}{\partial \mathbf{x}_{p}}\left(\mathbf{x}^{K L}\right) & =\frac{\partial \mathbf{F}_{q}^{K L}}{\partial \mathbf{x}_{p}}\left(\mathbf{x}^{K L}\right) \mathbf{F}_{q}^{K L, P, n^{-1}}
\end{aligned}
$$

Note that when calculating the generalized force in Equation (32)(33), $\mathbf{F}^{K L, E \operatorname{tr}}$ is used even though the associated stress may not satisfy the yield criteria. This is a consequence of the variational FEM discretization of the analogous formula for the stress in terms of derivative of the strain energy density[Bonet and Wood 2008]. We provide the calculation of $\mathbf{F}_{q}^{K L}\left(\mathbf{x}^{K L}\right)$ and $\frac{\partial \mathrm{F}_{q}^{K L}}{\partial \mathbf{x}_{p}}\left(\mathbf{x}^{K L}\right)$ in the supplementary technical document [Guo et al. 2018].

\section{MPM DISCRETIZATION}

We use MPM to discretize our elastoplastic model for frictional contact. We represent the shell using particles connected with subd interpolation as in $\S 9$. That is, we consider the subd FEM control point as particles in an MPM method. This allows us to resolve contact and collision automatically through the elastoplastic constitutive behavior when we transfer to the background grid. There is no need for any collision detection or resolution other than that inherent in the MPM discretization of the continuum model. Furthermore, our approach naturally allows for coupling with materials (e.g. granular sand, snow and soil) simulated with MPM.

MPM is a hybrid Lagrangian/Eulerian approach. However, the primary representation of material for MPM is the Lagrangian state. 
At time $t^{n}$, we store particle position $\mathrm{x}_{p}^{n}$, velocity $\mathbf{v}_{p}^{n}$, initial mass $m_{p}$, initial volume $V_{p}^{0}$, affine velocity $\mathrm{C}_{p}^{n}$ for all materials in the simulation. Similar to Jiang et al.[2017], we classify particles as either: (i) traditional MPM particles, (ii) subd particles or (iii) continuum shell shearing/compression particles. Particles of type (i) are used for coupling with traditional MPM materials like sand or snow. Types (ii) and (iii) are associated with elasticity and frictional contact respectively in the subd shell mesh. Furthermore, particles of type ( $i i)$ are control vertices in $\mathbf{x}^{K L}$ (see §9) for the subd shell and particles of type (iii) are quadrature points for the shearing component of the energy in Equation (10) and lie on the subd surface. For particles of type $(i)$, we store the elastic deformation gradient $\mathrm{F}_{p}^{E, n}$. For particles of type (iii), we store the time $t^{n}$ elastic shearing $\mathbf{a}_{p 3}^{E}$ and the parameters in the mid-surface $\left(\xi_{p 1}, \xi_{p 2}\right)$ associated with the particle. As in Jiang et al.[2017], we use the notation $\mathcal{I}^{(i)}, \mathcal{I}^{(i i)}, \mathcal{I}^{(i i i)}$ to represent the sets of particle indices of types (i), (ii) and (iii) respectively. At each of the quadrature points used in the Kirchhoff-Love energy, we store the deformation gradient and its elastic and plastic components $\mathrm{F}_{q}^{K L, n}, \mathrm{~F}_{q}^{K L, E, n}, \mathrm{~F}_{q}^{K L, P, n}$, the reference contravariant basis vectors $\overline{\mathrm{g}}_{q}^{i}$ needed for deformation gradient computation, and the mid-surface parameters $\left(\xi_{p 1}, \xi_{p 2}\right)$ associated with the point. Although these quadrature points are not MPM particles and are not used in transfers to and from the grid etc., we additionally use $\mathcal{I}^{(\mathrm{iv})}$ to denote the collection of quadrature points used in the Kirchhoff-Love energy. We illustrate all particle and quadrature point types in Figure 11.

In MPM, the Eulerian grid can be viewed as an auxiliary structure for updating the Lagrangian state. We first transfer the particle mass and momentum state to an equivalent grid counterpart. We use $m_{\mathrm{i}}^{n}$ to denote the mass of Eulerian grid node $\mathbf{x}_{\mathbf{i}}$ at time $t^{n}, \mathbf{v}_{\mathbf{i}}^{n}$ to denote its velocity and $\mathbf{p}_{\mathbf{i}}^{n+1}$ to denote its linear momentum after the grid update. The grid momentum is updated from the force defined as the gradient of the potential energy with respect to grid node motion. The motion of the grid is then interpolated to the particles to update the Lagrangian state without ever actually moving grid nodes. Our approach is ultimately very similar to other MPM methods that define forces from a notion of potential energy [Daviet and BertailsDescoubes 2016; Jiang et al. 2015; Klár et al. 2016; Stomakhin et al. 2013; Yue et al. 2015] and particularly Jiang et al.[2017]. We briefly discuss aspects common to the approach of Jiang et al.[2017] and discuss our novel modifications needed for subd shells in more detail. We summarize essential steps in the algorithm below.

(1) Transfer to grid: Transfer mass and momentum from particles to grid. $§ 10.1$

(2) Update grid momentum: Update grid momentum from potential-energy-based and body forces.

(3) Transfer to particles: Transfer velocities from grid to particles. $\$ 10.3$

(4) Update positions and trial elastic state: Update particle position, deformation gradient and trial elastic state assuming no plasticity over the time step. $\S 10.4$

(5) Update plasticity: Project trial elastic and plastic deformation gradients for plasticity return mapping. $§ 10.5$

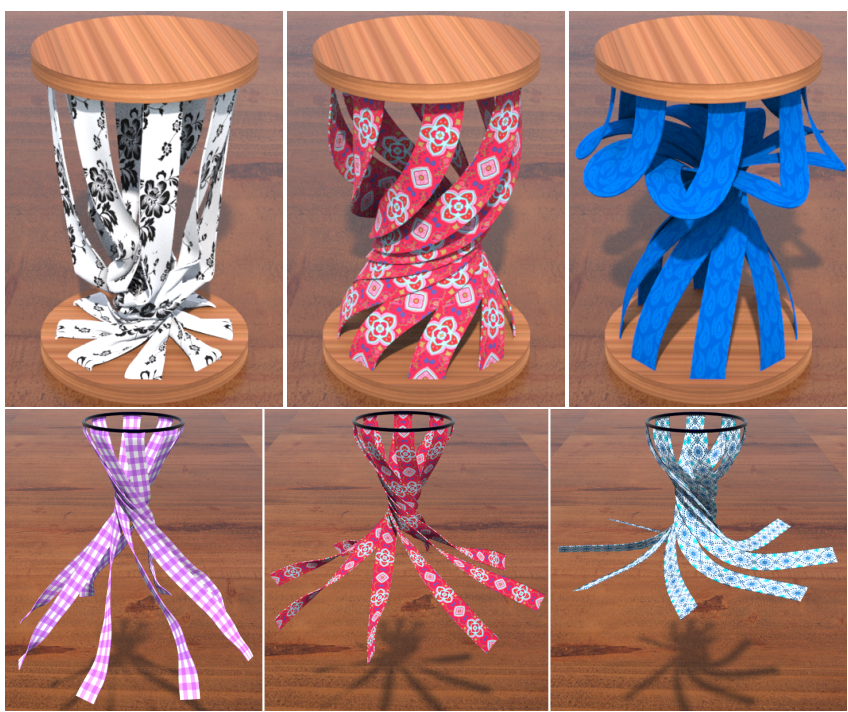

Fig. 12. Ribbons. We illustrate interesting dynamics achieved from colliding ribbons with increasing thickness (from left to right).

\subsection{Grid Transfers: Particle to Grid}

To update the Lagrangian state, we transfer mass and momentum from particles $\mathbf{x}_{p}^{n}$ to the grid nodes $\mathbf{x}_{\mathbf{i}}$ using APIC [Jiang et al. 2015].

$$
\begin{aligned}
m_{\mathrm{i}}^{n} & =\sum_{p} w_{\mathrm{i} p}^{n} m_{p} \\
\mathbf{v}_{\mathbf{i}}^{n} & =\frac{1}{m_{\mathrm{i}}^{n}} \sum_{p} w_{i p}^{n} m_{p}\left(\mathbf{v}_{p}^{n}+\mathrm{C}_{p}^{n}\left(\mathbf{x}_{\mathbf{i}}^{n}-\mathbf{x}_{p}^{n}\right)\right)
\end{aligned}
$$

Here $w_{\mathbf{i} p}^{n}=N\left(\mathbf{x}_{p}^{n}-\mathbf{x}_{\mathbf{i}}\right)$ is the weight of interaction between particle $\mathbf{x}_{p}^{n}$ and grid node $\mathbf{x}_{\mathbf{i}}$. The $N(\mathbf{x})$ are linear, quadratic or cubic B-spline kernels used for interpolation over the grid. $\mathrm{v}_{p}^{n}$ and $\mathrm{C}_{p}^{n}$ define an affine notion of velocity local to the particle.

\subsection{Grid Momentum Update}

The grid momentum update uses the updated Lagrangian view of the governing physics [Belytschko et al. 2013; Fu et al. 2017]. The grid at time $t^{n}$, after transferring state from the Lagrangian particles, is an alternative Lagrangian mesh with degrees of freedom $\mathbf{x}_{\mathbf{i}}, \mathbf{v}_{\mathbf{i}}^{n}$ and mass $m_{\mathbf{i}}^{n}$. Its update is derived from the Lagrangian FEM discretization of a problem with a notion of potential energy. The internal force is the negative gradient of the potential energy with respect to positional changes. Using $\mathrm{x}_{\mathrm{i}}^{n+1}$ and $\mathrm{p}_{\mathrm{i}}^{n+1}$ to denote the new position and linear momentum state after the time step, the grid discretization has the form

$$
\begin{aligned}
& \mathbf{x}_{\mathrm{i}}^{n+1}=\mathbf{x}_{\mathbf{i}}+\frac{\Delta t}{m_{\mathbf{i}}^{n}} \mathbf{p}_{\mathrm{i}}^{n+1} \\
& \mathbf{p}_{\mathbf{i}}^{n+1}=m_{\mathrm{i}}^{n} \mathbf{v}_{\mathbf{i}}^{n}-\Delta t \frac{\partial \Psi}{\partial \mathbf{x}_{\mathbf{i}}}\left(\mathbf{x}^{*}\right)+\Delta t m_{\mathrm{i}}^{n} \mathbf{g}
\end{aligned}
$$

where $\Psi(\mathbf{x})$ is the potential energy which depends on the positional state where we use $\mathbf{x}^{*}=\left(\mathbf{x}_{\mathbf{i}^{1}}^{*}, \mathbf{x}_{\mathbf{i}^{2}}^{*}, \ldots\right)^{T}$ to denote the vector of all grid node positions. In the case of symplectic Euler integration, 


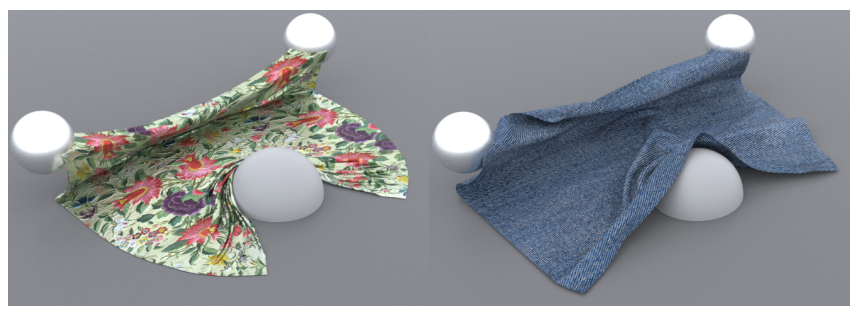

(a)

(b)

Fig. 13. Orthotropic Model. A range of materials can be simulated with our continuum shell formulation. Here we use the data-driven model of Clyde et al. [2017] for woven silk (left) and denim (right) materials. The model naturally allows for characteristic buckling and wrinkling behaviors in this object collision test.

$\mathbf{x}_{\mathbf{i}}^{*}=\mathbf{x}_{\mathbf{i}}$ and in the case of backward Euler, $\mathbf{x}_{\mathbf{i}}^{*}=\mathrm{x}_{\mathbf{i}}^{n+1}$. We note that the grid nodes are not actually moved from $x_{i}$ to $x_{i}^{n+1}$. Instead, the motion of the grid is interpolated to the particles (see $§ 10.3$ ).

The potential energy $\Psi$ is a sum of the contributions from the shell $\Psi^{C S}$ and from traditional MPM particles $\psi^{M}$ used for coupling multiple materials.

$$
\begin{gathered}
\Psi\left(\mathbf{x}^{*}\right)=\sum_{p \in \mathcal{I}^{(i)}} \psi^{M}\left(\mathbf{F}_{p}^{E, \operatorname{tr}}\left(\mathbf{x}^{*}\right)\right) V_{p}^{0}+\Psi^{C S}\left(\mathbf{x}^{*}\right) \\
\Psi^{C S}\left(\mathbf{x}^{*}\right)=\sum_{p \in \mathcal{I}^{(i i i)}} \chi\left(\mathbf{a}_{p \alpha}\left(\mathbf{x}^{K L}\left(\mathbf{x}^{*}\right)\right) \otimes \overline{\mathbf{a}}_{p \alpha}\right. \\
\left.+\mathbf{a}_{p 3}^{E, \operatorname{tr}}\left(\mathbf{x}^{K L}\left(\mathbf{x}^{*}\right)\right) \otimes \overline{\mathbf{a}}_{p 3}\right) V_{p}^{0} \\
+\sum_{q \in I^{(i v)}} \psi\left(\mathbf{F}_{q}^{K L, E \operatorname{tr}}\left(\mathbf{x}^{K L}\left(\mathbf{x}^{*}\right)\right)\right) V_{q}^{0} .
\end{gathered}
$$

Here $\psi^{M}$ is the contribution from the standard MPM potential discretization (see e.g. Stomakhin et al.[2013]) and $\Psi^{C S}$ is the contribution from the continuum shell. An advantage of the MPM approach is that coupling is achieved between any materials whose constitutive behaviors can be defined from potential energies. With any such models, coupling is achieved by first representing the motion of the materials in a Lagrangian way (e.g. discrete particles or Lagrangian meshes) and defining their motion and the way it effects their potential energy in terms of interpolation from the grid. With this model, coupling is a simple as defining the total potential energy as the sum of the varied materials.

The energy $\Psi^{C S}$ is the sum of the discretization of the KirchhoffLove component in Equation (10) given in Equation (31) and the frictional contact energy in Equation (23) obtained from the quadrature points $q \in \mathcal{I}^{(i v)}$ and $p \in \mathcal{I}^{(i i i)}$ respectively. We highlight the dependence of these potentials on the grid motion $\mathrm{x}^{*}$. For particles of type $(i)$, this dependence follows from the updated Lagrangian formulation

$$
\mathbf{F}_{p}^{E, \operatorname{tr}}\left(\mathbf{x}^{*}\right)=\left(\sum_{\mathbf{i}} \mathbf{x}_{\mathbf{i}}^{*} \otimes \nabla w_{\mathbf{i} p}^{n}\right) \mathbf{F}_{p}^{E, n}
$$

Here $\nabla w_{\mathbf{i} p}^{n}=\nabla N\left(\mathbf{x}_{p}^{n}-\mathbf{x}_{\mathbf{i}}\right)$ is the gradient of the grid interpolating function (or weight gradient) and $\left(\sum_{\mathbf{i}} \mathbf{x}_{\mathbf{i}}^{*} \otimes \nabla w_{\mathbf{i} p}^{n}\right)$ represents deformation induced by the grid motion $\mathbf{x}^{*}$. For particles of type (iii), the dependence follows from the updated Lagrangian

$$
\mathbf{a}_{p 3}^{E, \operatorname{tr}}\left(\mathbf{x}^{*}\right)=\left(\sum_{\mathbf{i}} \mathbf{x}_{\mathbf{i}}^{*} \otimes \nabla w_{\mathbf{i} p}^{n}\right) \mathbf{a}_{p 3}^{E, n}
$$

and from interpolation the $\mathbf{x}^{K L}\left(\mathbf{x}^{*}\right)$ in Equation (45) in $\mathbf{a}_{p \alpha}\left(\mathbf{x}^{K L}\left(\mathbf{x}^{*}\right)\right)$ [Guo et al. 2018]. Following the approaches in Jiang et al.[2017; 2015], the mid-surface control points for the shell are interpolated from the grid degrees of freedom as

$$
\mathbf{x}_{p}^{*}=\sum_{\mathbf{i}} \mathbf{x}_{\mathbf{i}}^{*} w_{\mathbf{i} p}^{n}, \quad p \in \mathcal{I}^{(i i)} .
$$

This interpolation also affects the discrete Kirchhoff-Love term through quadrature points $q \in \mathcal{I}^{(i v)}$.

Taking the $\mathrm{x}^{*}$ dependence into account and using the chain rule, the potential energy based forces obtained from the gradient of $\Psi$ with respect to $\mathbf{x}^{*}$ are

$$
\begin{aligned}
\frac{\partial \Psi}{\partial \mathbf{x}_{\mathbf{i}}}\left(\mathbf{x}^{*}\right) & =\mathbf{f}_{\mathbf{i}}^{(i)}\left(\mathbf{x}^{*}\right)+\mathbf{f}_{\mathbf{i}}^{(i i)}\left(\mathbf{x}^{*}\right)+\mathbf{f}_{\mathbf{i}}^{(i i i)}\left(\mathbf{x}^{*}\right) \\
\mathbf{f}_{\mathbf{i}}^{(i)}\left(\mathbf{x}^{*}\right) & =\sum_{p \in \mathcal{I}^{(i)}} \frac{\partial \psi^{M}}{\partial \mathbf{F}^{E}}\left(\mathbf{F}_{p}^{E, \operatorname{tr}}\left(\mathbf{x}^{*}\right)\right) \mathbf{F}_{p}^{E, n^{T}} \nabla w_{\mathbf{i} p}^{n} V_{p}^{0} \\
\mathbf{f}_{\mathbf{i}}^{(i i)}\left(\mathbf{x}^{*}\right) & =\sum_{p \in \mathcal{I}^{(i i)}} w_{\mathrm{i} p}^{n} \mathbf{f}_{p}^{K L}\left(\mathbf{x}^{K L}\left(\mathbf{x}^{*}\right)\right) \\
\mathbf{f}_{\mathrm{i}}^{(i i i)}\left(\mathbf{x}^{*}\right) & =\sum_{p \in \mathcal{I}^{(i i i)}} \tau_{p}^{S} \tilde{\mathbf{a}}_{p}^{\beta}: \frac{\partial \mathbf{a}_{p \beta}}{\partial \mathbf{x}_{p}} w_{\mathrm{i} p}^{n}+\tau_{p}^{S} \tilde{\mathbf{a}}_{p}^{3}: \nabla w_{\mathbf{i} p}^{n} \mathbf{a}_{p 3}^{E, n}
\end{aligned}
$$

In Equation (48), $\mathrm{f}_{p}^{K L}$ is the generalized Kirchhoff-Love force from Equation (32). In Equation (49), the stress $\tau_{p}^{S}$ is from Equation (26) and the vector $\tilde{\mathbf{a}}_{p}^{3}$ is the third contravariant basis vector with respect to the covariant basis $\left\{\mathbf{a}_{\alpha}\left(\mathbf{x}^{*}\right), \mathbf{a}_{3}^{E, \operatorname{tr}}\left(\mathbf{x}^{*}\right)\right\}$. We refer to the supplementary technical document for this derivation [Guo et al. 2018].

\subsection{Grid Transfers: Grid to Particle}

The grid to particle transfer defines the time $t^{n+1}$ affine velocity local to particle $\mathbf{x}_{p}^{n}$ in terms of $\mathbf{v}_{p}^{n+1}$ and $\mathrm{C}_{p}^{n+1}$ from

$$
\begin{aligned}
\mathbf{v}_{p}^{n+1} & =\sum_{\mathbf{i}} w_{\mathbf{i} p}^{n} \frac{\mathbf{p}_{\mathbf{i}}^{n+1}}{m_{\mathbf{i}}^{n}} \\
\tilde{\mathbf{C}}_{p}^{n+1} & =\frac{12}{\Delta x^{2}(d+1)} \sum_{\mathbf{i}} w_{\mathbf{i} p}^{n} \frac{\mathbf{p}_{\mathbf{i}}^{n+1}}{m_{\mathbf{i}}^{n}} \otimes\left(\mathbf{x}_{\mathbf{i}}^{n}-\mathbf{x}_{p}^{n}\right) \\
\mathbf{C}_{p}^{n+1} & =(1-v) \tilde{\mathbf{C}}_{p}^{n+1}+\frac{v}{2}\left(\tilde{\mathbf{C}}_{p}^{n+1}-\tilde{\mathbf{C}}_{p}^{n+1 T}\right)
\end{aligned}
$$

Here $d$ is the B-spline degree ( $d=3$ for cubic b-spline interpolation, $d=2$ for quadratic B-spline interpolation) and $\Delta x$ is the Eulerian grid spacing. $v$ is the explicit damping coefficient from Jiang et al.[2017] where $v=0$ is completely undamped and $\frac{1}{2}\left(\tilde{\mathbf{C}}_{p}^{n+1}-\tilde{\mathrm{C}}_{p}^{n+1 T}\right)$ is the RPIC transfer from Jiang et al.[2015]. 


\subsection{Update Positions and Trial Elastic State}

For particles of type $(i)$ and (ii), positions are moved with the interpolated grid node velocities. For particles of type (iii), positions are updated based on interpolation from updated particles of type (ii).

$$
\begin{aligned}
& \mathrm{x}_{p}^{n+1}=\mathrm{x}_{p}^{n}+\Delta t \mathbf{v}_{p}^{n+1}=\sum_{\mathrm{i}} \mathrm{x}_{\mathrm{i}}^{n+1} w_{\mathrm{i} p}^{n}, p \in \mathcal{I}^{(i)} \cup \mathcal{I}^{(i i)} \\
& \mathrm{x}_{p}^{n+1}=\sum_{p^{(i i)} \in \mathcal{I}^{(i i)}} \mathrm{x}_{p^{(i i)}}^{n+1} N_{p^{(i i)}}^{S D}\left(\xi_{p 1}, \xi_{p 2}\right), p \in \mathcal{I}^{(i i i)}
\end{aligned}
$$

We first assume there was no additional plastic flow over the time step and consider a trial state of elastic deformation. For particles of type (i) and (iii), the trial elastic deformation $\mathbf{F}_{p}^{E, \operatorname{tr}}$ and $\mathbf{a}_{p 3}^{E \text {, tr }}$ are computed as in Equations (43) and (44) respectively with $\mathrm{x}_{\mathrm{i}}^{*}=\mathrm{x}_{\mathrm{i}}^{n+1}$ For Kirchhoff-Love quadrature points $q \in \mathcal{I}^{(i v)}$, the trial elastic deformation gradient $\mathrm{F}_{q}^{K L, E \text { tr }}$ is computed from Equation (35) where $\mathbf{x}^{K L}\left(\mathbf{x}^{*}\right)$ is interpolated as in Equation (45) with $\mathbf{x}_{\mathbf{i}}^{*}=\mathbf{x}_{\mathbf{i}}^{n+1}$.

\subsection{Update Plasticity}

The assumption of no plastic flow over the time step is often safe. However, if the trial state of elastic stresses are not inside the yield surfaces associated with denting, frictional contact, etc. then they must be projected to satisfy the constraint. For particles $p \in \mathcal{I}^{(i)}$, $\mathrm{F}_{p}^{E, \operatorname{tr}}$ is projected to $\mathrm{F}_{p}^{E, n+1}$ in accordance with whichever yield surface is being used (e.g. the Drucker-Prager law in Klár et al.[2016]). For quadrature points $q \in \mathcal{I}^{(i v)}, \mathrm{F}_{q}^{E \text {,tr }}$ and $\mathbf{F}_{q}^{P \text {,tr }}$ are projected to $\mathbf{F}_{q}^{E, n+1}$ and $\mathbf{F}_{q}^{P, n+1}$ in accordance with the denting return mapping in $\S 7.1$. Lastly, the $\mathbf{a}_{p 3}^{E \text {,tr }}$ are projected to $\mathbf{a}_{p 3}^{n+1}$ in accordance with the frictional contact return mapping in Equation (29).

\section{RESULTS}

We demonstrate the efficacy of our method on a number of representative examples that exhibit appreciable bending and persistent self-collision and show that our method automatically allows for coupling with granular materials. Furthermore, we demonstrate the range of behaviors that are possible with the parameters in our model. We list the runtime performance for all of our examples in Table 1. All simulations were run on an Intel Xeon E5-2687W v4 system with 48 hyperthreads and 128GB of RAM. We report the timing in terms of average seconds of computation per frame. We chose $\Delta t$ in an adaptive manner that is restricted by a CFL condition when the particle velocities are high. In all of our simulations we use a CFL number equal to 0.3, i.e., we do not allow particles to move further than $0.3 \Delta x$ in a time step.

\subsection{Effect of Shell Thickness}

We control the bending stiffness of the shell by varying the thickness $\tau$. In Figure 1, six cylinders with increasing thickness from left to right free-fall and drop on the ground. In Figure 5, four cylinders of decreasing thickness from left to right buckle under lateral pressure and exhibit characteristic buckling patterns. In Figure 12, ribbons of varying thickness are planted in plates and twisted to produce interesting buckling phenomena.

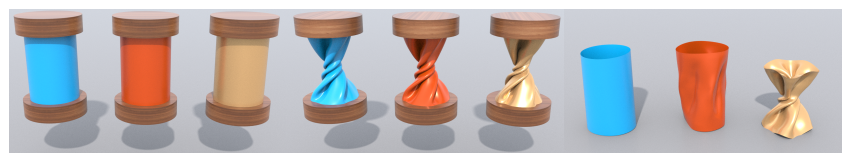

Fig. 14. Plastic shell deformation. The effect of the yield condition in Equation (13) is shown here with decreasing values of the coefficient $c_{v M}$ (from left to right). Larger values correspond to a larger stress needed for before denting plasticity is induced. The cylinders are twisted and then dropped to the ground to illustrate the plastic deformation.

\subsection{Woven Fabrics}

We demonstrate that our method supports any potential function in the Kirchhoff-Love shell model. In particular, we implement the datadriven orthotropic model for woven fabrics from Clyde et al.[2017] with parameters fitted from experimental data. In Figure. $4 \mathrm{a}$ and $4 \mathrm{~b}$, we twist and compress sleeves made of denim and silk. In Figure. 13a and $13 \mathrm{~b}$, we suspend squares of silk and denim which then collide with moving spheres. Our model accurately captures the behaviors of these real-world materials.

\subsection{Self Collisions}

Our model successfully resolves self-collision without any use of collision detection or constraint modeling outside the MPM discretization. We demonstrate this in a number of representative scenarios. In Figure 2, the spheres and the diving boards, both modeled as shells, collide with each other. In Figure 1 and Figure 3, we demonstrate self-collisions resolution for clothing simulation stress tests. In Figure 9, four decks of cards collide and then slide against each other to demonstrate the effect of varying friction coefficients.

\subsection{Plasticity for Denting}

Our method naturally incorporates the effect of plasticity in the shell. In Figure 14, three cylinders with different yield stress are twisted and then released. By changing the yield stress, we are able to control the amount of denting. In Figure 8, a square sheet of metal is compressed and then dented with a rod. The effect of plasticity creates permanent buckling and denting deformation.

\subsection{Two-way Coupling}

Our MPM approach automatically resolves coupling of different materials. In Figure 1, a cup is filled with slush and then released and toppled. The cup is modeled as a shell and the slush is modeled as in Stomakhin et al.[2013]. This example demonstrates that our method successfully resolves the interactions between two different materials of millions of particles with moderate computation cost.

\subsection{Resolution Refinement}

In Figure 10 we examine the behavior of our method under refinement of grid and subd mesh spatial resolution. This refinement study is done on a sleeve-buckling simulation with boundary conditions compressing the material at top and bottom. As the spatial resolution is increased, the simulation converges to the characteristic buckling pattern that is expected. 


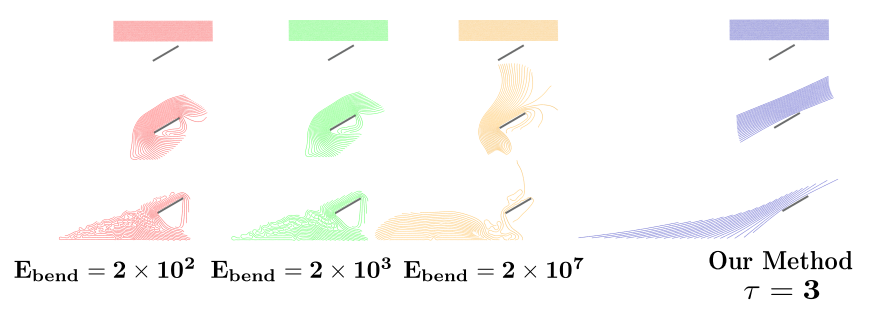

Fig. 15. Jiang et al. [2017] comparison. We demonstrate that only moderate bending is possible with the approach of Jiang et al. [2017]. Our approach allows for a much wider range of bending resistance.

\subsection{Bending with Jiang et al.}

We demonstrate the failure of the Jiang et al. [2017] model in achieving significant bending resistance. In Figure 15 we compare our model with the Jiang et al. generalized to bending with the addition of bending springs. The frictional contact model in Jiang et al. [2017] was not designed for bending resistance, however, it is possible to simply add bending cross springs to their model even though it violates the stress assumptions. We show that this is not capable of generating significant resistance to bending whereas our approach is designed to support stiff shells and thin membranes.

\section{DISCUSSION AND LIMITATIONS}

While our method can efficiently simulate thin shells with extreme contact and collision, there are a number of notable limitations. First, we have the same artifacts as Jiang et al. [2017], namely visible separation if $\Delta x$ is too large, persistent wrinkles if subd mesh resolution is too high relative to the grid resolution and self-penetration if the resolution is too low relative to the grid (see Figure 16). Also, the time step size is generally smaller than those used for membranes in Jiang et al. [2017]. This is due to the added stiffness associated with shell thickness and bending. With MPM, the increase in time step size with implicit time stepping is bounded above since particles cannot move more than a grid cell in a time step without causing bunching, self-collision or material inversion. Therefore the demand on the efficiency of nonlinear solver for the implicit systems is very high. Unfortunately this demand is difficult to meet since the nonlinear systems have non-symmetric linearizations that

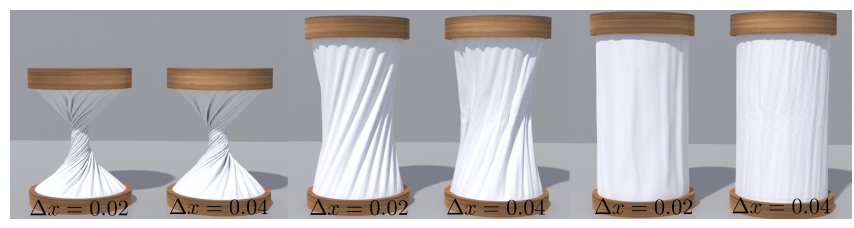

Fig. 16. Grid resolution dependent wrinkling. Our method suffers from persistent wrinkling if the subd mesh resolution is too high relative to the grid resolution. We demonstrate this phenomenon here with a cloth twisting comparison example. In both examples, the subd mesh $\Delta x=0.02$. The example on the left has grid $\Delta x=0.02$ whereas the one on the right has grid $\Delta x=0.04$. result from the plasticity [Klár et al. 2016]. "Lagging" the plasticity as in Stomakhin et al.[2013] provides a symmetric linearization but can cause cohesion artifacts that are unacceptable for frictional contact applications. Development of a solver that is more efficient than Newton's method with GMRES for the linearized systems is an interesting area of future work.

\section{ACKNOWLEDGMENTS}

The work is supported by NSF CCF-1422795, ONR (N000141110719, N000141210834), DOD (W81XWH-15-1-0147), Intel STC-Visual Computing Grant (20112360) as well as a gift from Disney Research.

\section{REFERENCES}

T. Belytschko, W. Liu, B. Moran, and K. Elkhodary. 2013. Nonlinear finite elements for continua and structures. John Wiley and sons.

J. Bonet and R. Wood. 2008. Nonlinear continuum mechanics for finite element analysis. Cambridge University Press.

R. Bridson, R. Fedkiw, and J. Anderson. 2002. Robust Treatment of Collisions, Contact and Friction for Cloth Animation. ACM Trans Graph 21, 3 (2002), 594-603. https: //doi.org/10.1145/566654.566623

R. Bridson, S. Marino, and R. Fedkiw. 2003. Simulation of Clothing with Folds and Wrinkles. In Proc ACM SIGGRAPH/Eurograph Symp Comp Anim (SCA '03). Eurographics Association, Aire-la-Ville, Switzerland, Switzerland, 28-36. https: //doi.org/10.2312/SCA03/028-036

Edwin Catmull and James Clark. 1978. Recursively generated B-spline surfaces on arbitrary topological meshes. Computer-aided design 10, 6 (1978), 350-355.

B. Chen and M. Govindaraj. 1995. A Physically Based Model of Fabric Drape Using Flexible Shell Theory. Text Res f 65, 6 (1995), 324-330. https://doi.org/10.1177/ 004051759506500603

F. Cirak and M. Ortiz. 2001. Fully C1-conforming subdivision elements for finite deformation thin-shell analysis. Int f Num Meth Eng 51, 7 (2001), 813-833. https: //doi.org/10.1002/nme.182

F. Cirak, M. Ortiz, and P. Schröder. 2000. Subdivision surfaces: a new paradigm for thin-shell finite-element analysis. Int f Num Meth Eng 47, 12 (2000), 2039-2072.

David Clyde. 2017. Numerical Subdivision Surfaces for Simulation and Data Driven Modeling of Woven Cloth. Ph.D. Dissertation. University of California, Los Angeles.

D. Clyde, J. Teran, and R. Tamstorf. 2017. Modeling and data-driven parameter estimation for woven fabrics. In Proc ACM SIGGRAPH / Eurograp Symp Comp Anim (SCA '17). ACM, New York, NY, USA, 17:1-17:11. https://doi.org/10.1145/3099564.3099577

J. Collier, B. Collier, G. O'Toole, and S. Sargand. 1991. Drape prediction by means of finite-element analysis. J Textile Inst 82, 1 (1991), 96-107. https://doi.org/10.1080/ 00405009108658741

G. Daviet and F. Bertails-Descoubes. 2016. A Semi-implicit Material Point Method for the Continuum Simulation of Granular Materials. ACM Trans Graph 35, 4 (2016), 102:1-102:13. https://doi.org/10.1145/2897824.2925877

R. Echter, B. Oesterle, and M. Bischoff. 2013. A hierarchic family of isogeometric shell finite elements. Comp Meth App Mech Eng 254 (2013), 170 - 180. https: //doi.org/10.1016/j.cma.2012.10.018

J. Eischen, S. Deng, and T. Clapp. 1996. Finite-element modeling and control of flexible fabric parts. IEEE Comp Graph App 16, 5 (1996), 71-80. https://doi.org/10.1109/38. 536277

Table 1. All simulations were run on Intel Xeon E5-2687W v4 system with 48 hyperthreads and $128 \mathrm{~GB}$ of RAM. Element \# denotes number of quadrilaterals. Particle \# denotes the number of type (i), (ii) and (iii) particles.

\begin{tabular}{lrrrrr}
\hline & Figure & seconds/frame & Element \# & Particle \# & $\Delta x$ \\
\hline Cup of Slush & 1 & 273 & $19.5 K$ & $3.1 M$ & 0.04 \\
Shirt Twister & 1 & 188 & $168 K$ & $504 K$ & 0.005 \\
Six Cylinders & 1 & $2 / 2 / 2 / 2 / 2 / 4$ & $20 K$ & $60 K$ & 0.025 \\
Walk Cycle & 1 & 75 & $33 K$ & $100 K$ & 10 \\
Spheres On Diving Board & 2 & 87 & $150 K$ & $450 K$ & 0.027 \\
Pants Twister & 3 & 78 & $131 K$ & $393 K$ & 0.005 \\
Silk Twister & $4 \mathrm{a}$ & $63 K$ & $315 K$ & 0.02 \\
Denim Twister & $4 \mathrm{~b}$ & 3 & $15.8 K$ & $47 K$ & 0.04 \\
Sleeves (Yellow) & 5 & 97 & $126 K$ & $378 K$ & 0.01 \\
Sleeves (Others) & 5 & 8 & $31 K$ & $93 K$ & 0.02 \\
Denting with Rod & 8 & 1 & $5 K$ & $15 K$ & 0.01 \\
Playing Cards & 9 & 55 & $23 K$ & $115 K$ & 0.02 \\
Fixed Ribbons & 12 & $3 / 8 / 30$ & $12 K$ & $93 K$ & 0.02 \\
Free Ribbons & 12 & $4 / 4 / 7$ & $12 K$ & $85 K$ & 0.02 \\
Silk Curtain & $13 \mathrm{a}$ & 167 & $75 K$ & $227 K$ & 0.004 \\
Denim Curtain & $13 \mathrm{~b}$ & 3 & $8 K$ & $25 K$ & 0.012 \\
Plastic Twister & 14 & $<1$ & $5 K$ & $14 K$ & 0.06 \\
\hline
\end{tabular}


O. Etzmuss, J. Gross, and W. Strasser. 2003a. Deriving a particle system from continuum mechanics for the animation of deformable objects. IEEE Trans Vis Comp Graph 9, 4 (Oct. 2003), 538-550. https://doi.org/10.1109/TVCG.2003.1260747

O. Etzmuss, M. Keckeisen, and W. Strasser. 2003b. A fast finite element solution for cloth modeling. In Proc 11th Pac Conf Comp Graph App (PG '03). IEEE Computer Society, Washington, DC, USA, 244-254. https://doi.org/10.1109/PCCGA.2003.1238266

Y. Fan, J. Litven, D. Levin, and D. Pai. 2013. Eulerian-on-Lagrangian Simulation. ACM Trans Graph 32, 3 (2013), 22:1-22:9. https://doi.org/10.1145/2487228.2487230

Y. Fan, J. Litven, and D. Pai. 2014. Active Volumetric Musculoskeletal Systems. ACM Trans Graph 33, 4 (2014), 152:1-152:9. https://doi.org/10.1145/2601097.2601215

C. Fu, Q. Guo, T. Gast, C. Jiang, and J. Teran. 2017. A Polynomial Particle-in-cell Method. ACM Trans Graph 36, 6 (Nov. 2017), 222:1-222:12. https://doi.org/10.1145/3130800. 3130878

L. Gan, N. Ly, and G. Steven. 1995. A study of fabric deformation using nonlinear finite elements. Text Res f 65, 11 (1995), 660-668. https://doi.org/10.1177/ 004051759506501106

Y. Gingold, A. Secord, J. Han, E. Grinspun, and D. Zorin. 2004. A discrete model for inelastic deformation of thin shells. In Tech Report: Courant Institute of Mathematical Sciences, New York University.

A. Golas, R. Narain, and M. Lin. 2014. Continuum modeling of crowd turbulence. Phys Rev E 90 (2014), 042816. Issue 4. https://doi.org/10.1103/PhysRevE.90.042816

O. Gonzalez and A. Stuart. 2008. A first course in continuum mechanics. Cambridge University Press.

E. Grinspun, F. Cirak, P. Schröder, and M. Ortiz. 1999. Non-linear mechanics and collisions for subdivision surfaces. Technical report, Caltech Multi-Res Modeling Group (1999)

E. Grinspun, A. Hirani, M. Desbrun, and P. Schröder. 2003. Discrete Shells. In Proc ACM SIGGRAPH/Eurograph Symp Comp Anim (SCA '03). Eurographics Association, Airela-Ville, Switzerland, Switzerland, 62-67. https://doi.org/10.2312/SCA03/062-067

E. Grinspun, P. Krysl, and P. Schröder. 2002. CHARMS: A simple framework for adaptive simulation. ACM Trans Graph 21, 3 (2002), 281-290. https://doi.org/10.1145/566654 566578

E. Grinspun and P. Schröder. 2001. Normal bounds for subdivision-surface interference detection. In IEEE Viz. 333-340. https://doi.org/10.1109/VISUAL.2001.964529

Q. Guo, X. Han, C. Fu, T. Gast, R. Tamstorf, and J. Teran. 2018. A Material Point Method for Thin Shells with Frictional Contact: Supplementary Technical Document. (2018).

D. Harmon, E. Vouga, R. Tamstorf, and E. Grinspun. 2008. Robust Treatment of Simultaneous Collisions. ACM Trans Graph 27, 3 (2008), 23:1-23:4. https://doi.org/10.1145/ 1360612.1360622

C. Jiang, T. Gast, and J. Teran. 2017. Anisotropic elastoplasticity for cloth, knit and hair frictional contact. ACM Trans Graph 36, 4 (2017). https://doi.org/10.1145/3072959. 3073623

C. Jiang, C. Schroeder, A. Selle, J. Teran, and A. Stomakhin. 2015. The Affine ParticleIn-Cell Method. ACM Trans Graph 34, 4 (2015), 51:1-51:10. https://doi.org/10.1145/ 2766996

C. Kane, E. Repetto, M. Ortiz, and J. Marsden. 1999. Finite element analysis of nonsmooth contact. Comp Meth App Mech Eng 180, 1 (1999), 1 - 26. https://doi.org/10.1016/ S0045-7825(99)00034-

P. Kaufmann, S. Martin, M. Botsch, and M. Gross. 2009. Implementation of discontinuous Galerkin Kirchhoff-Love shells. ETH Zurich, Department of Computer Science, Technical Report No. 622 (2009)

J. Kiendl, K. Bletzinger, J. Linhard, and R. Wuchner. 2009. Isogeometric shell analysis with Kirchhoff-Love elements. Comp Meth App Mech Eng 198, 49 (2009), 3902 - 3914 https://doi.org/10.1016/j.cma.2009.08.013

J. Kiendl, M. Hsu, M. Wu, and A. Reali. 2015. Isogeometric Kirchhoff-Love shell formulations for general hyperelastic materials. Comp Meth App Mech Eng 291, Supplement C (2015), 280-303. https://doi.org/10.1016/j.cma.2015.03.010

G. Klár, T. Gast, A. Pradhana, C. Fu, C. Schroeder, C. Jiang, and J. Teran. 2016. DruckerPrager Elastoplasticity for Sand Animation. ACM Trans Graph 35, 4 (2016), 103:1103:12. https://doi.org/10.1145/2897824.2925906

D. Levin, J. Litven, G. Jones, S. Sueda, and D. Pai. 2011. Eulerian Solid Simulation with Contact. ACM Trans Graph 30, 4 (2011), 36:1-36:10. https://doi.org/10.1145/2010324. 1964931

D. Li, S. Sueda, D. Neog, and D. Pai. 2013. Thin Skin Elastodynamics. ACM Trans Graph 32, 4 (2013), 49:1-49:10. https://doi.org/10.1145/2461912.2462008

Q. Long, P. Bornemann, and F. Cirak. 2012. Shear flexible subdivision shells. Int 7 Num Meth Eng 90, 13 (2012), 1549-1577. https://doi.org/10.1002/nme.3368

L. De Lorenzis, P. Wriggers, and T. Hughes. 2014. Isogeometric contact: a review. GAMM-Mitteilungen 37, 1 (2014), 85-123. https://doi.org/10.1002/gamm.201410005

J. Lu. 2011. Isogeometric contact analysis: Geometric basis and formulation for frictionless contact. Comp Meth App Mech Eng 200, 5 (2011), 726 - 741. https: //doi.org/10.1016/j.cma.2010.10.001

J. Lu and C. Zheng. 2014. Dynamic cloth simulation by isogeometric analysis. Comp Meth App Mech Eng 268, Supplement C (2014), 475 - 493. https://doi.org/10.1016/j. cma.2013.09.016
X. Man and C. Swan. 2007. A mathematical modeling framework for analysis of functional clothing. J Eng Fibers Fabrics 2, 3 (2007), 10-28. http://www.jeffjournal org/papers/Volume2/Swan(6-14R1).pdf

S. Martin, P. Kaufmann, M. Botsch, E. Grinspun, and M. Gross. 2010. Unified simulation of elastic rods, shells, and solids. ACM Trans Graph 29, 4 (2010), 39:1-39:10. https: //doi.org/10.1145/1778765.1778776

M. Matzen and M. Bischoff. 2016. A weighted point-based formulation for isogeometric contact. Comp Meth App Mech Eng 308, Supplement C (2016), 73-95. https://doi. org/10.1016/j.cma.2016.04.010

M. Matzen, T. Cichosz, and M. Bischoff. 2013. A point to segment contact formulation for isogeometric, NURBS based finite elements. Comp Meth App Mech Eng 255, Supplement C (2013), 27-39. https://doi.org/10.1016/j.cma.2012.11.011

A. McAdams, A. Selle, K. Ward, E. Sifakis, and J. Teran. 2009. Detail Preserving Continuum Simulation of Straight Hair. ACM Trans Graph 28, 3 (2009), 62:1-62:6. https://doi.org/10.1145/1531326.1531368

R. Mindlin. 1951. Influence of rotary inertia and shear on flexural motions of isotropic elastic plates. J App Mech 18 (1951), 31-38.

R. Narain, A. Golas, S. Curtis, and M. Lin. 2009. Aggregate Dynamics for Dense Crowd Simulation. ACM Trans Graph 28, 5 (2009), 122:1-122:8. https://doi.org/10.1145/ 1618452.1618468

R. Narain, A. Golas, and M. Lin. 2010. Free-flowing granular materials with two-way solid coupling. ACM Trans Graph 29, 6 (2010), 173:1-173:10. https://doi.org/10.1145/ 1882261.1866195

R. Narain, T. Pfaff, and J. O’Brien. 2013. Folding and crumpling adaptive sheets. ACM Trans Graph 32, 4 (July 2013), 51:1-51:8. https://doi.org/10.1145/2461912.2462010

L. Noels and R. Radovitzky. 2008. A new discontinuous Galerkin method for KirchhoffLove shells. Comp Meth App Mech Eng 197 (2008), 2901-2929. https://doi.org/10. 1016/j.cma.2008.01.018

M. Otaduy, R. Tamstorf, D. Steinemann, and M. Gross. 2009. Implicit Contact Handling for Deformable Objects. Comp Graph Forum 28, 2 (2009). https://doi.org/10.1111/j. 1467-8659.2009.01396.x

E. Sifakis, S. Marino, and J. Teran. 2008. Globally Coupled Collision Handling Using Volume Preserving Impulses. In Proc 2008 ACM SIGGRAPH/Eurographics Symp Comp Anim. 147-153. https://doi.org/10.2312/SCA/SCA08/147-153

J. Simo and D. Fox. 1989. On a stress resultant geometrically exact shell model. Part I Formulation and optimal parametrization. Comp Meth App Mech Eng 72, 3 (1989), 267 - 304. https://doi.org/10.1016/0045-7825(89)90002-9

Jos Stam. 1998. Exact evaluation of Catmull-Clark subdivision surfaces at arbitrary parameter values. In Proceedings of the 25th annual conference on Computer graphics and interactive techniques. ACM, 395-404.

A. Stomakhin, C. Schroeder, L. Chai, J. Teran, and A. Selle. 2013. A Material Point Method for snow simulation. ACM Trans Graph 32, 4 (2013), 102:1-102:10. https: //doi.org/10.1145/2461912.2461948

M. Tang, H. Wang, L. Tang, R. Tong, and D. Manocha. 2016. CAMA: Contact-Aware Matrix Assembly with Unified Collision Handling for GPU-based Cloth Simulation. Comp Graph Forum 35, 2 (2016), 511-521. https://doi.org/10.1111/cgf.12851

A. Temizer, P. Wriggers, and T. Hughes. 2011. Contact treatment in isogeometric analysis with NURBS. Comp Meth App Mech Eng 200, 9 (2011), 1100 - 1112. https: //doi.org/10.1016/j.cma.2010.11.020

Y. Teng, D. Levin, and T. Kim. 2016. Eulerian Solid-fluid Coupling. ACM Trans Graph 35, 6 (2016), 200:1-200:8. https://doi.org/10.1145/2980179.2980229

D. Terzopoulos, J. Platt, A. Barr, and K. Fleischer. 1987. Elastically Deformable Models. SIGGRAPH Comput Graph 21, 4 (1987), 205-214. https://doi.org/10.1145/37402.37427

B. Thomaszewski, M. Wacker, and W. Strasser. 2006. A consistent bending model for cloth simulation with corotational subdivision finite elements. In Proc ACM SIGGRAPH/Eurograph Symp Comp Anim. Eurographics Association, 107-116. https: //doi.org/10.2312/SCA/SCA06/107-116

A. Wawrzinek, K. Hildebrandt, and K. Polthier. 2011. Koiter's thin shells on CatmullClark limit surfaces. In Vision, Modeling, and Visualization (2011), Peter Eisert, Joachim Hornegger, and Konrad Polthier (Eds.). The Eurographics Association. https://doi.org/10.2312/PE/VMV/VMV11/113-120

Y. Yue, B. Smith, C. Batty, C. Zheng, and E. Grinspun. 2015. Continuum foam: a material point method for shear-dependent flows. ACM Trans Graph 34, 5 (2015), 160:1-160:20. https://doi.org/10.1145/2751541

Y. Zhu and R. Bridson. 2005. Animating sand as a fluid. ACM Trans Graph 24, 3 (2005), 965-972. https://doi.org/10.1145/1073204.1073298 